4

\title{
A pigment-deficient mosquito line with fluorescent salivary glands enables in vivo imaging of individual Plasmodium sporozoites
}

\author{
Dennis Klug ${ }^{1, \#}$, Katharina Arnold ${ }^{1,2}$, Eric Marois ${ }^{1}$ and Stéphanie A. Blandin ${ }^{1}$ \\ ${ }^{1}$ Université de Strasbourg, CNRS UPR9022, INSERM U1257, Institut de Biologie \\ Moléculaire et Cellulaire, 67000 Strasbourg, France \\ Germany \\ ${ }^{2}$ Current address: German Cancer Research Institute (DKFZ), 69120 Heidelberg, \\ \# to whom correspondence should be addressed: \\ dklug@unistra.fr
}

\section{Abstract}

Plasmodium parasites are the causative agent of malaria, a major health burden in subSaharan Africa. A key step in the transmission process of Plasmodium is the colonization of the salivary glands of the female Anopheles mosquito by the parasite sporozoite stage. How sporozoites recognize and invade the salivary glands is still poorly understood. Here we generated salivary gland reporter lines in the African malaria mosquito Anopheles coluzzii using salivary gland-specific promoters of the genes encoding anopheline antiplatelet protein (AAPP), the triple functional domain protein (TRIO) and saglin (SAG). The observed expression pattern of the DsRed and roGFP2 fluorescent reporters revealed lobe-specific activity of these promoters within the salivary glands, restricted either to the distal lobes or the middle lobe. We characterized four mosquito lines (AAPP-DsRed, AAPP-roGFP2, TRIODsRed and SAG-EGFP) in terms of localization, expression strength and onset of expression, as well as potential influences of the expressed fluorescent reporters on the infection with Plasmodium berghei and salivary gland morphology. Furthermore, using crosses with a pigmentation deficient yellow(-) mosquito line, we demonstrate that our salivary gland reporter lines represent a valuable tool to study the process of salivary gland colonization by Plasmodium parasites in live mosquitoes. 


\section{Introduction}

Vector borne diseases have been a major health burden in the history of mankind. An efficient way to counteract diseases transmitted by blood sucking arthropods is to prevent transmission itself by reducing the number of vectors in the environment or by preventing vectors from biting humans in risk areas. As a consequence of malaria control interventions, approximately 663 million clinical malaria cases could be averted between 2000 and 2015. The most effective measure turned out to be the use of insecticide treated bet nets which prevented an estimated $68 \%$ of cases (Bhatt et al., 2015). Still, preventive measures are counteracted by the very effective transmission of vector borne pathogens, which hampers eradication approaches. This is especially true for the causative agent of malaria, a protist named Plasmodium, which evolved highly specific adaptations to ensure efficient transmission between mosquitoes and human.

After infection of the mosquito midgut through the uptake of a blood meal from an infected host, Plasmodium parasites form a cyst, called oocyst, below the basal lamina of the midgut epithelium where they undergo multiple rounds of DNA replication. Rapid cell division, called schizogony, then follows, leading to the generation of several thousand of sporozoites per oocyst. These cells are released from the cysts and travel from the midgut to the salivary glands and, after being deposited in the skin during a blood meal, traverse the dermis, enter blood capillaries and reach the human liver. The sporozoites' distinctive cell polarization and crescent shape, in combination with high motility, allows them to „drill“ through tissues. In addition, sporozoites are highly deformable enabling passing through densely packed environments. An important feature of sporozoites is their long survival time, up to several weeks, in mosquito tissues (Mayne, 1922).

While it is believed that the invasion process of the salivary glands by sporozoites that have egressed from oocysts is rapid ( 1-10 minutes), in vivo data supporting this assumption is lacking (Douglas et al., 2015). Still, the time a sporozoite needs to reach and successfully invade the glands is an important parameter, because at this timepoint, a mosquito is considered infective to humans. Moreover, the very quick invasion of the salivary glands suggests that sporozoites have specific receptors that recognize ligands exposed on the gland surface. Up to date only two mosquito proteins, saglin and circumsporozoite protein (CSP) binding protein (CSPBP), have been described to support salivary gland invasion of sporozoites (Ghosh and Jacobs-Lorena, 2009; Wang et al., 2013). Knockdown experiments of both proteins have shown that sporozoite numbers in the salivary glands are reduced 
68

compared to controls, indicating a lower invasion rate. However, overexpression of saglin in the distal lobes of Anopheles stephensi did not lead to an increase in the number of salivary gland resident sporozoites, and immunofluorescence-staining using anti-saglin antibodies indicated that this protein is expressed in the middle lobe of each salivary gland (O'Brochta et $a l ., 2019)$. This observation is puzzling since sporozoites strongly prefer to invade the distal lobes, with sporozoite invasion of the smaller middle lobe being less prominent or even completely absent (Wells and Andrew, 2019). Thus, the relation between saglin and sporozoites may be more complex than a simple ligand-receptor interaction. Microscopic studies of infected glands revealed that sporozoites form large accumulations in organized „stacks“ inside cavities of acinar cells. The astonishing degree of order of these structures raises the question whether sporozoites can recognize each other. Alternatively, specifically preferred invasion sites may lead to accumulations at these entry points. However, since receptor-ligand interactions between sporozoites and acinar cells are still undefined, no specific entry site has been identified thus far.

Proteins secreted in the saliva of blood-sucking arthropods have been a long-term focus of interest. Saliva proteins often possess vasodilating, anti-coagulating and anti-inflammatory properties which counteract host responses and enable efficient blood feeding (Ribeiro and Francischetti, 2003). One protein specifically found in Anopheles gambiae saliva is TRIO (triple functional domain protein) that shares low homology to Drosophila melanogaster TRIO (Francischetti et al., 2002, Arcà et al., 2005, Dragovic et al., 2018). Interestingly TRIO expression increases in the presence of P. berghei sporozoites (Dragovic et al., 2018). The anopheline antiplatelet protein (AAPP) has anti-thrombotic functions and prevents collageninduced platelet aggregation (Yoshida et al., 2008; Hayashi et al., 2012). Consistently, transgenic co-expression of an AAPP-specific single chain antibody blocking AAPP function negatively affects blood feeding in vivo (Islam et al., 2019). The region upstream of the aapp gene was the first promoter used to drive salivary gland-specific transgenes in the Asian malaria mosquito Anopheles stephensi (Yoshida and Watanabe, 2006). Here we compare the promoter activities of the regions upstream of the aapp, trio and saglin genes in African Anopheles coluzzii mosquitoes and we test potential applications of the generated reporter lines to investigate sporozoite migration. 


\section{Results}

Generation of transgenic mosquito lines with salivary gland-specific reporter expression and low pigmentation

To generate salivary gland reporter lines, the 5' upstream sequences of saglin (AGAP000610), aapp (AGAP009974) and trio (AGAP001374) were used to drive the expression of DsRed and hGrx1-roGFP2 from transgenic constructs integrated in the X1 docking line (Fig. 1A, Fig. S1) (Volohonsky et al., 2015). The fluorescence reporter hGrx1-roGFP2 was chosen because it bears the advantages of EGFP and enables the measurement of the oxidation level of the intracellular glutathione pool by ratiometric quantification (Gutscher et al., 2008). We used the aapp promoter sequence from Anopheles coluzzii orthologous to the one used to drive salivary gland-specific transgene expression in Anopheles stephensi (Yoshida and Watanabe, 2006). For both saglin and trio, we defined putative promoter regions according to the genomic context. In addition, as the selected saglin promoter region was very short $(220 \mathrm{bp}$,

113 Fig. S2), we also used CRISPR/Cas9 to integrate a fluorescence cassette encoding EGFP into 114 the endogenous saglin locus, replacing the coding sequence, to study saglin promoter activity in its native genomic context. For this, site directed knock-in mutagenesis was performed by injecting a plasmid encoding three guide RNAs targeting saglin and carrying a repair template marked with $3 x P 3$-EGFP into mosquito eggs from a line with germline-specific expression of

118 Cas9 (vasa-Cas9), generating a sag(-)KI line (Fig. 1B, S3A). To place the EGFP gene 119 directly under control of the saglin promoter, the 3xP3 promoter used to selected transgenic 120 mosquito larvae was flanked by lox sites to enable its excision by Cre recombination (Fig. 121 S3E, Material \& Methods). Similarly, we generated a knockin (KI) into the yellow gene that 122 encodes a protein required for the synthesis of black pigments in insects (Hunt and Bridges, 123 1916; Wittkopp, True and Carroll, 2002). For this, we used a plasmid with guide RNAs 124 specific for yellow and corresponding homology arms flanking a fluorescence cassette 125 expressing EGFP surrounded by lox sites (Fig. S3B). Through crossing of salivary gland 126 reporter lines with low pigmented yellow(-)KI mosquitoes, we aimed to improve the

127 fluorescence signal of the expressed reporter and extend the range of applications, e.g. for in 128 vivo imaging. Genotyping by PCR confirmed the successful generation of the salivary gland 129 reporter lines aapp-DsRed, aapp-hGrxl-roGFP2, trio-DsRed and sag-DsRed (Fig S1) as well 130 as the mosquito lines $\operatorname{sag}(-) K I$ and yellow(-)KI (Fig S3C,D). Loss of the $3 x P 3$ promoter in 131 sag(-)EX mosquitoes (mosquitoes lacking the $3 x P 3$ promoter after $C r e$ mediated excision) 
132 was confirmed by PCR in the progeny of a cross between the sag(-)KI line and a mosquito

133 line expressing Cre recombinase in the germline (Fig. S3E) (Volohonsky et al., 2015).

\section{Lobe-specific transcriptional activation by sag, aapp and trio promoter sequences}

136 Dissection of female salivary glands revealed a highly specific expression pattern of DsRed,

137 EGFP and hGrx1-roGFP2 driven by sag, aapp and trio promoter sequences inside salivary 138 glands (Fig. 1C). While no fluorescence was observed for DsRed driven by the cloned 5' 139 upstream sequence of the saglin gene in dissected salivary glands (Fig. S3A), promoter 140 activity was detected in the line where EGFP replaced the saglin gene (Fig. 1C), indicating 141 that essential transcriptional elements localize outside of the intergenic sequence between AGAP000609 and AGAP000610 (saglin) (Fig. S3B). Interestingly, fluorescence signals in 143 glands of female aapp-DsRed, aapp-hGrx1-roGFP2 mosquitoes were restricted to the distal 144 lobes, while female trio-DsRed and sag(-)EX mosquitoes displayed fluorescence exclusively 145 in the middle lobe (Fig. 1C). This difference became especially evident when crossing aapp146 hGrxl-roGFP2 mosquitoes with trio-DsRed mosquitoes giving rise to a trans-heterozygous offspring displaying hGrx1-roGFP2 expression in distal lobes combined with DsRed expression in the middle lobe (Fig. 1C). Similarly, when crossing aapp and trio DsRed reporter lines, the entire salivary gland was red fluorescent in the F1 progeny (Fig. 1C). Expression of trio-DsRed and aapp-DsRed was so strong that the respective parts of the salivary gland appeared pink colored even in brightfield microscopy (Fig. 1C). Of note, fluorescent reporter expression in the salivary glands of aapp-DsRed, aapp-hGrxl-roGFP2 and trio-DsRed mosquito colonies was restricted to females while no fluorescence signal could be observed in this organ in males (Fig. S4A). Imaging of aapp-DsRedP, aapp-hGrxlroGFP2 and trio-DsRed pupae during development revealed no distinct fluorescence signal in the head part where the salivary glands are located (Fig. S4B). Still, DsRed expression was visible in the salivary glands of aapp-DsRed females that had been dissected directly after emergence (Fig. 2A), indicating that the aapp promoter is already active in pharate adults. In contrast, no DsRed expression was observed in the glands of freshly hatched trio-DsRed females (Fig. 2A). To evaluate the expression profile over time we performed a time-course experiment by dissecting and imaging salivary glands of trio-DsRed and aapp-DsRed females every day. DsRed expression guided by aapp and trio promoters increases steadily until day 5

163 after hatching (Fig. 2B). The strongest increase in fluorescence in aapp-DsRed females was 164 observed from day three to four, and from day four to five in trio-DsRed females (Fig. 2B). The increase in DsRed in the salivary glands of aapp-DsRed and trio-DsRed females could 
166 also be followed in transmitted light based on the pink coloration of the distal lobes (Fig.

167 S4C). In Anopheles stephensi, AAPP expression was shown by qRT-PCR to be upregulated

168 upon blood feeding (Yoshida and Watanabe, 2006). We followed DsRed fluorescence levels

169 in the salivary glands of aapp-DsRed and trio-DsRed sibling mosquitoes that were fed on

170 blood or kept on sugar (Fig. 2C). Our results confirm that the aapp promoter is induced upon

171 ingestion of a blood meal while there was no difference between blood-fed and sugar-fed in

172 females expressing the trio-DsRed transgene.

Subcellular localization of fluorescent reporters and impact on salivary gland

176 During first microscopic examinations using low magnification, different intracellular 177 localizations of the fluorescent proteins DsRed, EGFP and hGrx1-roGFP2 were observed (Fig. 178 1C), which was confirmed by confocal microscopy. DsRed expressed in the salivary glands 179 of aapp-DsRed and trio-DsRed mosquitoes appeared spotty and localized in vesicles within 180 the cytoplasm of the acinar cells, while hGrx1-roGFP2 and EGFP expressed in the glands of aapp-hGrx1-roGFP2 and sag(-)KI mosquitoes displayed a cytoplasmic localization (Fig. 3A). Due to the anatomy of the secretory cells, which form an inward cavity into which saliva is secreted, the DsRed-positive vesicles localize mainly at the outer rim of the salivary gland. In contrast, localization of hGrx1-roGFP2 and EGFP was homogenous in the cytoplasm and appeared to be also nuclear (Fig. 3A). We also observed morphological alterations. While salivary glands of aapp-DsRed, trio-DsRed and sag(-)KI females were indistinguishable from wild type (G3), the apex of the distal lobes in aapp-hGrxl-roGFP2 expressing salivary glands appeared shrunken and we detected a strangulation between the apex and the remainder of the lobe (Fig. 3B,C). Acinar cells in the apex of the lobes that displayed the highest expression levels of hGrx1-roGFP2, appeared thinned and atrophied, and presented an inflation of the salivary duct in the apex region (Fig. 3A).

\section{Natural variation of DsRed expression in trio-DsRed mosquitoes}

194 During the generation of the salivary gland reporter lines and their subsequent outcrossing we observed that most L1 mosquito larvae carrying the trio-DsRed transgene displayed DsRed expression in the eyes (Fig. S5A), which was absent in the two other reporter lines aappDsRed and aapp-hGrxl-roGFP2. Interestingly, a few L4 stage larvae of the trio-DsRed line even displayed strong ubiquitous DsRed expression (Fig. S5A). At the pupal stage, DsRed expression was detected in the ocellus, an eye spot close to the main compound eye, the 
antennae and/or throughout the body. The observed expression patterns were highly variable and ranged from completely non-fluorescent pupae to pupae expressing DsRed in all three tissues (Fig. S4B, S6A). In adult mosquitoes, DsRed expression was less distinct compared to pupae although complete body fluorescence was occasionally observed in both males and females, with the strongest expression of DsRed in the salivary glands of females and the palps and antennae of males (Fig. S5B). Dissections of adult mosquitoes displaying whole body fluorescence at the pupal stage revealed strong and sex-independent DsRed signals in Malphigian tubules, an insect organ that performs functions similar to the vertebrate kidney (Fig. S5C). Of note, fluorescence in tubules strictly correlated to the high pupal fluorescence phenotype and was never observed in mosquitoes hatched from low fluorescent pupae (Fig. S5D). We next investigated sex-specificity of each fluorescence pattern at the pupal stage and inheritance of the high and low fluorescent trio-DsRed transgene. Non-fluorescent pupae were always females, while fluorescent antennae were observed exclusively in male pupae (Fig. S6B). In contrast, the presence of a fluorescent ocellus or body fluorescence was sexindependent (Fig. S6B). Quantification of the observed expression pattern revealed that a fluorescent ocellus, either alone or in combination with body fluorescence, was most frequently observed (Fig. S6C). The ratio of pupae that displayed body fluorescence to those that did not was close to $50 \%$ (Fig S6C). To follow the inheritance pattern of high and low body fluorescence, pupae were separated according to their fluorescence phenotype and intercrossed. Quantification of pupal DsRed expression in the F1 generation revealed a strong enrichment for whole body fluorescence $(\sim 80 \%)$ if high pupal body fluorescence was selected in the previous generation. In contrast, no pupae with high body fluorescence were observed in the offspring of mosquitoes hatched from low fluorescent pupae (Fig. S6D). This result indicates a form of Mendelian inheritance, with the locus conferring high body fluorescence being dominant over that conferring low body fluorescence. To investigate this further, virgin female pupae of the F1 generation displaying high pupal DsRed expression were crossed with wild type males. Ten single female families were created which were all screened for pupal body fluorescence. All families except family 8 displayed a 1:1 distribution for high and low pupal body fluorescence (Fig. S6E). Family 8 displayed exclusively high pupal body fluorescence, indicating that the founder female was homozygous for the locus conferring high body fluorescence, while all other females were heterozygous for the „low“ and the „high“ trio-DsRed loci. Notably, we detected high DsRed signals in the salivary glands of all adult females in families 5, 6, 7, 8 and 10 (other families were not investigated), regardless of whether individuals hatched from pupae displaying high or low body fluorescence. 
234 Genotyping of females homozygous for low trio-DsRed or heterozygous for WT/high trio-

235 DsRed revealed integration of the transgene into the X1 locus as a single copy (Fig. S7).

\section{Impact of reporter expression on infection with Plasmodium berghei}

238 To test if the expression of a fluorescent reporter in the salivary glands alters parasite 239 development in the mosquito, we infected the three reporter lines trio-DsRed, aapp-DsRed 240 and aapp-hGrxl-roGFP2 as well as the parental line G3 with the rodent malaria parasite

241 Plasmodium berghei line $\Delta$ p230p-GFP that constitutively expresses EGFP at high levels in all 242 developmental stages (Manzoni et al., 2015). Parasite counts observed in reporter lines varied 243 from 118 to 142 oocysts and were not significantly different from the control (121 oocysts) 244 (Fig. 4A). The average number of salivary gland sporozoites at day 17-18 after infection was 2458.200 to 17.400 sporozoites per mosquito in salivary gland reporter lines vs 9.600 in G3 246 controls (Fig. 4B). An average of 10.000 sporozoites is what is usually observed in our 247 laboratory, while numbers can fluctuate from 5.000 to more than 20.000 sporozoites per 248 mosquito even in non-transgenic lines, indicating no significant difference between the G3 249 control and reporter lines. To normalize the counts of salivary gland sporozoites relative to 250 differences in oocyst numbers, we calculated the number of sporozoites that had successfully 251 invaded the salivary glands per oocyst. Again, no significant difference was detected between 252 reporter lines (86-146 sporozoites per oocyst) and G3 control (133) mosquitoes (Fig. 4C). 253 These results indicate that fluorescence expression in the salivary glands does not affect 254 parasite development in mosquitoes or salivary gland infection with sporozoites.

Salivary gland reporter lines as a tool to investigate sporozoite biology / invasion of the salivary glands

258 We next investigated whether our highly fluorescent salivary gland reporter lines could facilitate in vivo imaging of Plasmodium development through the cuticle of a living mosquito using the same $P$. berghei line $\triangle p 230 p-G F P$ (Fig. 5A). This line is bright enough to make parasites visible through the mosquito cuticle, allowing pre-sorting of infected mosquitoes based on fluorescence in the mosquito midgut, the salivary glands or in the wing joint, a localization where hemolymph sporozoites tend to accumulate. Still, light absorption

264 by the pigmented cuticle and light scattering by chitin generate signal losses reducing image quality and resolution. To reduce light absorption, we crossed aapp-DsRed mosquitoes, displaying highly fluorescent salivary glands without altered morphology, with yellow(-)KI 267 mosquitoes, a loss-of-function line with low cuticle pigmentation (Fig. 5A). Yellow is a 
268 protein required for the synthesis of black pigments in insects, its absence renders the cuticle 269 pale and yellowish (Hunt and Bridges, 1916; Wittkopp, True and Carroll, 2002). As expected, 270 yellow(-)-aapp-DsRed mosquitoes displayed a low pigmented cuticle in combination with 271 highly fluorescent salivary glands (Fig. 5B). The decrease in pigmentation greatly increased 272 the observed GFP signal visible through the cuticle, notably in strongly pigmented areas that 273 appear almost black in WT mosquitoes (Fig. 5C). We next imaged mosquitoes infected with 274 P. berghei $\triangle p 230 p-G F P$ between day 17 and day 20 after infection, when sporozoites have 275 already invaded the salivary glands. In addition, mosquitoes were pre-sorted for GFP 276 fluorescence in the salivary glands (Fig. S8A) as well as in the wing joint (not shown) to 277 confirm the presence of sporozoites. While pre-sorting, we observed that the salivary glands 278 in a subset of mosquitoes (less than 1 out of 5) localized very close to the cuticle (Fig. S8B,C).

279 To facilitate in vivo imaging, we further selected mosquitoes positive for sporozoites and with 280 cuticular localization of at least one salivary gland. Subsequently the legs of selected 281 mosquitoes were removed and mosquitoes were glued on a microscopy slide. To prevent 282 squeezing of mosquitoes, cover slips were prepared with small plasticine feet at each corner, 283 and fixed with nail polish to prevent shifting of the cover against the microscopy slide (Fig. 284 S8D). Confocal imaging enabled to visualize precisely single sporozoites through the cuticle close to the salivary gland as well as subcellular structures, like the cavities of acinar cells, of the gland itself (Fig. 6A). Live imaging revealed that a considerable number of sporozoites persist in tissues close to the gland and sometimes perform active movement (Fig. 6B,C, Movies 1 and 2). We also observed single sporozoites moving actively through tissues in a 289 back and forth gliding manner (Fig. 6B, Movie 3) or making contact with the salivary gland although no invasion event could be monitored (Fig, 6D).

\section{Discussion}

293 Mosquito salivary glands have long interested entomologists, parasitologists, virologists and 294 microbiologists because they are a key organ in the transmission of vector borne diseases. 295 Still, the host-pathogen interactions that allow the specific recognition and colonization of this 296 organ by different pathogens has been understudied, probably in part because of the relative 297 difficulty to access and visualize these processes in comparison to midgut invasion. The 298 reporter lines we generated in this study represent valuable tools to circumvent some of these 299 issues: they allow (1) to easily visualize the glands, even in brightfield or DIC microscopy, 
(2) to distinguish distal and middle lobes of the salivary glands, and (3) to image single sporozoites in proximity to the salivary glands in vivo.

302 We used the promoters of three salivary gland genes, aapp, trio and saglin, to drive 303 fluorescent reporter expression in A. coluzzii mosquitoes. The different fluorescence patterns 304 obtained in the salivary glands of these lines show that the proteomes of middle and distal lobes are diverse and most likely contribute different saliva components. In addition, the variable fluorescence intensity in the distal lobes of both aapp lines (stronger towards the apex) point towards an even more complex expression pattern along the lobe. Interestingly the trio and the saglin promoters were only active in the middle lobe, which, for saglin, is coherent with immunofluorescence studies performed in A. stephensi (O'Brochta et al., 2019). This observation is puzzling since both proteins have been shown to either influence salivary gland colonization or transmission of Plasmodium sporozoites (Ghosh and Jacobs-Lorena, 2009; Dragovic et al., 2018) while parasites prefer to invade the distal lobes (Wells and Andrew, 2019) that lack activity of saglin and trio promoters. A possible explanation is that the site of expression of both proteins is not the site where they interact with parasites. The absence of reporter expression in mosquitoes expressing DsRed controlled by the saglin 5' upstream sequence is likely due to the shortness of the cloned promoter sequence. The saglin gene localizes in the middle of an array of four genes believed to be salivary glandspecific (Francischetti et al., 2002) and separated by short intergenic regions (Fig. S1). Regulatory sequences controlling transcription are likely located upstream and/or downstream of this array and might be shared by all four genes. Notably, the reporter lines display different subcellular localizations of hGrx1-roGFP2, EGFP and DsRed. While all expressed fluorescence, proteins lack any targeting signal which could explain a different localization, only hGrx1-roGFP2 and EGFP localized within the cytoplasm.

324 In contrast, DsRed displayed a vesicular pattern in both middle (trio) and distal lobes (aapp), while a DsRed2 variant driven by the aapp promoter in A. stephensi mosquitoes was shown to be cytoplasmic (Yoshida and Watanabe, 2006). The difference in localization between DsRed and other fluorescent reporters points to the presence of a cryptic trafficking signal in DsRed recognized by the acinar cells of $A$. coluzzii. Indeed, although we did not investigate their nature, we also observed the presence of vesicles in the salivary glands of wild type mosquitoes with similar distribution and morphology and displaying weak red autofluorescence (Fig. S3A). DsRed could potentially be trafficked to this or a similar compartment explaining its unique localization. Differences in salivary gland morphology between aapp-DsRed and aapp-hGrxl-roGFP2 expressing mosquitoes are likely due to the 
334 expression of human Glutaredoxin 1 (hGrx1) and not of roGFP2, a protein derived from

335 EGFP with 2 amino acid changes. Indeed, no change in morphology was detected in sag(-)EX

336 mosquitoes expressing EGFP alone in the middle lobe. Still, EGFP appeared less expressed in

337 the sag(-)EX compared to hGrx1-roGFP2 in aapp-hGrx1-roGFP2 (Fig. 1) and we cannot

338 exclude that the acinar cells of the middle and distal lobes react differently to the expression

339 of the respective reporters or that the cells show different responses to roGFP2 and EGFP.

340 Despite its influence on cell morphology the salivary glands of hGrx1-roGFP2 expressing

341 mosquitoes retained their integrity even with high mosquito age and apoptosis of cells

342 expressing the fluorescent reporter was not observed.

343 We also report a surprisingly large panel of fluorescence patterns in mosquitoes expressing 344 the trio-DsRed transgene. The trio promoter activated transcription of DsRed in the salivary 345 glands of all females (but not in males), but also in ocelli, antennae and Malpighian tubules as 346 well as whole body in some but not all mosquitoes, independently of their sex and with a 347 strict correlation between strong DsRed expression in pupal body and adult Malpighian 348 tubules. The fact that trio expression was reported to be salivary gland-specific (Dragovic et $a l ., 2018)$ suggests that the region we selected upstream of trio does not contain all regulatory elements that control its natural expression, or that the genomic context of the X1 locus may influence its expression. Up to date, the number of available promoters able to drive ubiquitous expression of transgenes in Anopheles mosquitoes is limited (Adolfi et al., 2018), thus replacing DsRed in a selected highly fluorescent line could provide a new means to ubiquitously express transgenes. By sorting and crossing according to the observed pupal 355 fluorescence phenotype, it was possible to separate alleles conferring high and low pupal 356 body fluorescence, which allowed the production of a colony enriched with the high pupal 357 fluorescence phenotype and a colony showing only low pupal body fluorescence, especially in the ocelli. This observation pointed towards a genetically encoded difference which is inherited in a Mendelian manner rather than a transient (epi)genetic modification. Genotyping confirmed the presence of a single copy of the transgene in the X1 locus in all types of mosquitoes. Since fluorescent ocelli, antennae and whole body expression were only observed in the trio-DsRed population, we propose that the different DsRed expression patterns are 363 locus-specific effects caused by interactions between the selected trio promoter sequence and 364 regulatory elements within sequences flanking the transgene, or in trans. The transgene integration into the X1 locus may also have occurred with slight alterations that could influence DsRed expression or a subset of mosquitoes may have inherited a second copy of 367 the transgene integrated in a different locus causing different locus-specific expression effects. 
368 However, DsRed positive ocelli and antennae were observed in both high and low 369 fluorescence populations, which is most likely explained by a variable DsRed expression from the transgene inserted at X1. While the observed DsRed expression patterns in larvae 371 and pupae are helpful in the laboratory to select transgenic individuals it also urges caution in 372 interpreting results as it clearly shows that transcription is contextual.

373 Intravital imaging has revealed the dynamics of parasite development inside its vertebrate 374 host (Sturm et al., 2006; Wang et al., 2020). While optical windows in mice is a well 375 implemented method (De Niz et al., 2019), in vivo imaging through the cuticle of insects is 376 scarcely performed, mostly because it is difficult to achieve good resolution by imaging 377 through the insect cuticle that scatters and absorbs light very efficiently. Recent studies have explored the possibility to circumvent this issue through the application of clearing protocols on infected mosquitoes to improve resolution and enable imaging through whole mosquitoes (de Niz et al., 2019; Mori, Hirai and Mita, 2019). However, while these improvements are remarkable, imaging of cleared specimens only represents snapshots whereas mapping of dynamic processes would be desired. Imaging of tissues ex vivo in combination with the use of hemolymph-like media allows the observation of dynamic processes (Klug and 384 Frischknecht, 2017). Still, imaging ex vivo samples can only be performed for a limited amount of time and artifacts due to the artificial tissue environment cannot be excluded. A step forward towards intravital imaging was made recently by developing a protocol that combines feeding of dyes relevant for imaging with blood feeding prior to sample preparation (Trisnadi and Barillas-Mury, 2020). Due to the inflation of the mosquito abdomen directly after feeding, cuticle plates open up and create temporary optical windows that can be exploited for microscopy. Still, in this case, mosquitoes were decapitated and tightly flattened between microscopy slide and cover slip, thus allowing for optimal imaging but maintaining in vivo conditions only for a limited time. To demonstrate the use of the salivary gland reporter lines for imaging purposes, we crossed aapp-DsRed mosquitoes with the low pigmented mosquito line yellow(-)KI deficient for black pigment synthesis. In a subset of mosquitoes, salivary glands localized very closely to the cuticle allowing the recognition of subcellular structures. Combining brighter cuticle with cuticular positioning of the salivary glands within the mosquito thorax was instrumental to achieve visualization of single sporozoites through the cuticle and we were able to get an insight into sporozoite behaviour in vivo. It is generally believed that sporozoites, after they have been released from the oocyst, are passively transported to the salivary glands and engage in active motility only upon contact with acinar cells (Douglas et al., 2015). Our in vivo observations indicate that 
sporozoites can perform active motility even when not in contact with the salivary gland

403 (Movie 1, 2 and 3). Interestingly, a significant number of sporozoites appeared trapped in 404 close proximity to the gland. These sporozoite accumulations are already known from other sites of the mosquito circulatory system (Hillyer, Barreau and Vernick, 2007). Thus sporozoite localization in the salivary gland area, for example through the detection of GFP, does not necessarily represent a measure for successfully invaded sporozoites (Fig. 6A). Here we provide a proof of concept of in vivo salivary gland imaging, and we hope that the method can be further improved to gain a better insight of the invasion of salivary glands by sporozoites. The injection of fluorescent beads or dyes could be used as vitality measure to monitor the heartbeat of the mosquito while, at the same time, highlighting contact areas with hemolymph and salivary glands. Also we did not exhaust the repertoire of possible genetic modifications to decrease pigmentation of the cuticle. Deletion of the genes tan and ebony, two enzymes which regulate the homeostasis of the pigment precursors dopamine and N- $\beta-$ alanyldopamine, in combination with the deletion of yellow could potentially further decrease cuticle pigmentation (Wittkopp, True and Carroll, 2002; True et al., 2005). Another possibility could be the overexpression of arylalkylamine- $N$-acetyltransferase, an enzyme converting dopamine into the sclerotizing precursor molecule $\mathrm{N}$-acetyldopamine, which was shown to affect pigmentation in different insect species by enhancing the production of colorless components of the cuticle (Osanai-Futahashi et al., 2012). A key improvement for in vivo imaging would be an increase in the number of mosquitoes displaying ideally positioned salivary glands. Preliminary monitoring experiments showed that the position of the gland does not significantly change over time in adult mosquitoes indicating that positioning occurs during metamorphosis. Should salivary gland positioning be genetically determined, selective breeding may help to improve the ratio of mosquitoes with cuticular salivary glands. Taken together we hope that the transgenic lines developed in this study can contribute to improve in vivo imaging and salivary gland dissections in Anopheles mosquitoes, and enable real-time imaging of Plasmodium and other vector borne pathogens.

\section{Materials \& methods}

\section{Animals}

433 Transgenesis was performed in the Anopheles coluzzii strain X1 (Volohonsky et al., 2015) or 434 in a strain expressing Cas9 from the X1 locus under control of the vasa promoter 
(transgenesis plasmid: Addgene \# 173670) and introgressed into the Ngousso genetic

436 background. Wild type Anopheles coluzzii strains Ngousso and G3 were used as controls.

437 CD1 mice purchased from Charles River or from our own colonies were used for both mosquito maintenance through blood meals, and for parasite maintenance and mosquito infections. For infections with rodent malaria parasites, we made use of the Plasmodium berghei line $\Delta p 230 p-G F P$ derived from the wild type background P. berghei ANKA (Manzoni et al., 2015).

\section{Breeding of mosquitoes}

444 Anopheles coluzzii mosquitoes were kept in standard conditions $\left(27^{\circ} \mathrm{C}, 75-80 \%\right.$ humidity, 12 $445 \mathrm{hr} / 12-\mathrm{hr}$ light/dark cycle). Larvae were hatched in pans of osmosed water and fed with 446 pulverized fish food (TetraMin, Melle, Germany). Adult mosquitoes were maintained on a $44710 \%$ sucrose solution. To propagate colonies, four to seven day old mosquitoes were blood 448 fed for 10-15 minutes on anesthetized mice and allowed to lay eggs on wet filter paper 2-3 449 days later. For mosquito blood feeding, female CD-1 mice ( $>35 \mathrm{~g}$ ) were anesthetized with a mixture of Zoletil (42.5 mg/kg) and Rompun $(8.5 \mathrm{mg} / \mathrm{kg})$ in $0.9 \% \mathrm{NaCl}$ solution.

\section{Construction of salivary gland reporter transgenes}

453 The 5'upstream sequences of saglin (primers P9 and P10), aapp (primers P1 and P2) and trio 454 (primers P7 and P8) were amplified from genomic DNA of $G 3$ mosquitoes isolated with the 455 Blood \& Tissue Kit (Qiagen) as shown in File S1. DsRed (primers P5 and P6 for aapp/trio; 456 P11 and P12 for saglin) and hGrx1-roGFP2 (primers P3 and P4) were amplified from the 457 plasmids pJET-DsRed-SV40 and pDSAP_actin-hGrx1-roGFP2 (donated by Raquel Mela458 Lopez). Primers are shown in Table S1. PCR was performed using Phusion polymerase according to the manufacturer recommendations (ThermoFisher Scientific) and subcloned in 460 the pJET vector using the CloneJET PCR Cloning Kit (ThermoFisher Scientific). The 5'upstream sequence of the saglin gene was fused to the DsRed gene by a Phusion mediated assembly PCR (TerMaat et al., 2009) before cloning into pJET. After sequence verification,

463 assembly blocks were fused into the destination vector pDSAP (Volohonsky et al., 2015, 464 Addgene \# 62293) using Goldengate cloning and generating pDSAP-aapp-DsRed, pDSAPaapp-hGrx1-roGFP2, pDSAP-trio-DsRed and pDSAP-saglin-DsRed. 


\section{Construct cloning for site-directed mutagenesis into yellow and saglin genes}

469 For the design of guide RNAs (gRNAs), the coding sequence of saglin as well as the first exon of the yellow gene were manually searched for PAMs (NGG), with $\mathrm{N}$ representing any nucleotide. Candidate gRNA sequences were investigated for potential off-target effects using http://www.rgenome.net/cas-designer/ (Cas-OFFinder) (Bae, Park and Kim, 2014). We selected gRNAs starting with a $\mathrm{G}$ considering the transcription start from the U6 promoter and differing by $\geq 3$ nucleotides from putative off-target sequences in the $A$. coluzzii genome.

475 For each knockin construct, linkers encoding the protospacer motif of three gRNAs (Table

476 S1) were generated by annealing two primers ordered from IDT DNA and cloned using $B b s I$ into pKSB-sgRNA (Addgene \#173671-3) vectors containing the U6 snRNA polymerase III promoter (AGAP013557), the invariable sequence of a standard crRNA-tracerRNA fusion (Jinek et al., 2012), and the RNA polIII TTTTT terminator. The three complete gRNAs blocks were then assembled into a single pKSB-sgRNA destination plasmid by GoldenGate cloning using different but matching BsaI overhangs. For the construction of repair templates, regions of homology on the 5' side (saglin: primers P29 and P30; yellow: primers P19 and P20) and on the 3' side (saglin: primers P31 and P32; yellow: primers P21 and P22) of the region targeted by the gRNAs were amplified from Ngousso genomic DNA and subcloned in the pJET vector, generating plasmids pJET-5'saglin, pJET-5'yellow, pJET-3'saglin and pJET-3'yellow. After sequence validation, all building blocks were fused in the correct order in the destination plasmid pENTR using GoldenGate assembly together with a building block containing either the fluorescence cassette pKSB-Lox3xP3Lox-EGFP or pKSB-Lox3xP3EGFP-Lox, generating pENTR-Saglin;Lox3xP3Lox-EGFP and pENTR-Yellow;Lox3xP3EGFP-Lox. Primers are shown in Table S1.

\section{Transgenesis of $A$. coluzzii}

493 All plasmids used for DNA injections were purified using Endofree kits (Qiagen). Freshly 494 laid eggs of the docking line $\mathrm{X} 1$ or the vasa-Cas 9 line were injected as described (Fuchs, 495 Nolan and Crisanti, 2013; Pondeville et al., 2014; Volohonsky et al., 2015). For ФC31496 integrase mediated transgensis a mix of three plasmids (pDSAP-aapp-DsRed, pDSAP-aapp497 hGrx1-roGFP2 and pDSAP-trio-DsRed $)(\sim 160 \mathrm{ng} / \mu \mathrm{L})$ and the integrase helper plasmid carrying the Vasa2-ФC31-Integrase gene (Addgene \# 62299, 200 ng/ $\mu \mathrm{L}$ ) diluted in 0.5x phosphate buffered saline ( $0.1 \mathrm{mM}$ NaHPO4 buffer, $5 \mathrm{mM} \mathrm{KCl}, \mathrm{pH} 6.8)$ was injected into approximately 500 embryos. The plasmid pDSAP-saglin-DsRed was injected in the same way but into a different batch of eggs. For site-directed knock-in mediated by Cas9, the 
502 plasmid solution (pENTR-Saglin;Lox3xP3Lox-EGFP or pENTR-Yellow;Lox3xP3Lox-

503 EGFP) ( 200-300 ng/ $\mu \mathrm{L})$ was diluted in PBS supplemented with $1 \mu \mathrm{M}$ Scr7 to inhibit non-

504 homologous end joining. Each construct was injected in a separate batch of approximately

505500 eggs obtained from vasa-Cas 9 females.

Salivary gland reporter lines sag-DsRed, trio-DsRed, aapp-DsRed and aapp-hGrx1508 roGFP2

509 Following egg micro-injection, surviving pupae were sorted according to sex, hatched in separate cages (G0 generation) and crossed with 100 mosquitoes of the other sex from a mosquito line expressing CFP from the X1 docking site that was used as a balancer of the new, non-fluorescent but puromycin resistant transgenic insertions at the same locus. Neonate

513 larvae from this cross were treated with puromycin $(0.05 \mathrm{mg} / \mathrm{mL})$. G1 pupae were 514 subsequently sex sorted and crossed with CFP positive mosquitoes to expand the population.

515 Adult puromycin resistant G2 females were sorted according to the observed fluorescence 516 phenotype (DsRed or roGFP2) in their salivary glands, reflecting the expression of the trio517 DsRed, aapp-DsRed or aapp-hGrxl-roGFP2 transgenes and crossed to CFP balancer males.

518 For sag-DsRed females, sorting was not necessary, as transgenesis was performed separately 519 by injection of the pDSAP-saglin-DsRed plasmid alone. Puromycin-resistant G3 larvae were 520 raised, intercrossed and the G4 offspring was sorted by COPAS (Marois et al., 2012) for the absence of CFP to select lines homozygous for sag-DsRed, trio-DsRed, aapp-DsRed and aapp-hGrxl-roGFP2. Lines were then raised without puromycin treatment. Single females of all generated colonies were genotyped by PCR to monitor correct integration of the transgene into the docking site (Fig. S1A,B). Successfully genotyped mosquito colonies were expanded and used to perform experiments.

\section{yellow(-)KI and sag(-)KI lines}

528 Pupae obtained from plasmid injected eggs were sorted according to sex and crossed with 100 529 Ngousso wild type partners. G1 neonate larvae were screened for the expression of EGFP 530 using a Nikon SMZ18 fluorescence Stereomicroscope. Selected transgenic larvae were sex 531 sorted at the pupal stage and crossed with an excess of wild type partners. The G2 progenies

532 (from a single positive G1 female for sag(-)KI and from 10 positive individuals for yellow($533(K I)$ were COPAS sorted to enrich for the integrated transgene and to remove the vasa-Cas 9 534 transgene (marked with DsRed). The generated sag(-)KI colony was kept in equilibrium with 535 the wild type allele, while the yellow(-)KI colony was completely homozygotized for the 
536 transgene by selecting for mosquitoes with a yellow body color. Homozygous sag(-)KI

537 mosquitoes were crossed with the C2S line expressing Cre in the germline (Volohonsky et al.,

538 2015) for Cre recombinase-mediated excision of the lox cassette to generate $\operatorname{sag}(-) E X$

539 mosquitoes.

540

\section{Genotyping of mosquito lines}

542 Genotyping was performed on genomic DNA extracted from single mosquitoes of aapp-

543 DsRed, aapp-hGrxl-roGFP2, trio-DsRed, sag(-)KI, sag(-)EX and yellow(-)KI colonies.

544 Genotyping PCRs were performed using GoTaq Green Mastermix (Promega) (Fig. S1B),

545 Phusion polymerase (ThermoFisher Scientific) or the Phire Tissue Direct PCR Master Mix

546 (ThermoFisher Scientific) (Fig. S2 and S7) according to manufacturer recommendations. See

547 Table $\mathbf{S 2}$ for genotyping primers.

\section{Mosquito infection and evaluation of parasite numbers}

550 Parasites were maintained as frozen stocks at $-80^{\circ} \mathrm{C}$ or by passage between mice. For this,

551 blood was taken by heart puncture from a donor mouse with a parasitaemia of 3-5\%, diluted

552 to $2.10^{7}$ parasitized erythrocytes in $100 \mu \mathrm{L} 0.9 \% \mathrm{NaCl}$ that were injected intravenously into a

553 naïve mouse. Parasitemia was monitored via flow cytometry (Accuri C6, BD biosciences)

554 three days later. Mosquitoes were fed for 10-15 min on anesthetized mice with $2.5-3.5 \%$

555 parasitemia. Blood fed females were sorted and kept on $10 \%$ sucrose at $21^{\circ} \mathrm{C}$ and $50-70 \%$

556 humidity. Midguts and salivary glands were dissected in PBS under a SZM-2 Zoom

557 Trinocular stereomicroscope (Optika). Images were acquired with the Nikon SMZ18

558 Stereomicroscope (without coverslip) or with a Zeiss LSM 780 confocal microscope (sealed

559 coverslip). To count oocysts, midguts were dissected 7-8 days post infection. Fluorescence

560 images of infected midguts were processed using the watershed segmentation plugin (Soille

561 and Vincent, 1990) and oocysts were subsequently counted using the ,analyze

562 particles“ function implemented in Fiji (Schindelin et al., 2012). To count salivary gland

563 sporozoites, salivary glands were collected in PBS 17-18 days post infection and ground with

564 a plastic pestle for one minute. The number of sporozoites was measured using a Neubauer

565 hemocytometer under a light microscope (Zeiss). 


\section{Fluorescence imaging, fluorescence measurements and image analysis}

570 Samples imaged using the Nikon SMZ18 Stereomicroscope were usually acquired using the respective fluorescence filter to visualize expression of DsRed or roGFP2/EGFP combined with brightfield to visualize the whole specimen. Images were taken using a 63-fold

573 magnification objective (NA 1.4) on a Zeiss LSM 780 confocal microscope (Fig. 3A), a 574 Zeiss Axiovert 200 fluorescence microscope (Fig. S2A) or using an iPhone 6 for the mounted 575 mosquitoes prepared for microscopy in Fig. S8D. All other images as well as images used for

576 fluorescence measurements were acquired with a Nikon SMZ18 Stereomicroscope. For 577 fluorescence quantifications, images were acquired in the DsRed channel and settings were 578 kept the same for all experiments. For each salivary gland, only a single lobe was measured 579 by analyzing a square of $51 \times 51$ pixels covering the central part of the fluorescent area to 580 determine the mean fluorescence intensity. The image processing for all experiments was 581 performed with Fiji (Schindelin et al., 2012).

\section{In vivo imaging}

584 For in vivo imaging experiments, infected yellow(-)-aapp-DsRed mosquitoes were prescreened between days $17-21$ post infection for the presence of hemolymph sporozoites (hinge of the wing, GFP fluorescence) and salivary gland sporozoites (salivary gland, GFP fluorescence) as well as for cuticular positioning of the salivary gland (DsRed signal) (Fig. S8B). After removing their legs, mosquitoes were glued on a microscopy slide using small amounts of UV-light reactive glue (Bondic Pocket) and covered with a coverslip prepared with plasticine feet at each corner to prevent a squeezing of the sample. Corners of the cover slip were coated with nail polish to prevent sliding. Imaging was performed on a Zeiss LSM 780 confocal microscope with GaAsP detector and Metamorph acquisition software.

\section{Statistical analysis}

595 Statistical analysis was performed using GraphPad Prism 5.0 (GraphPad, San Diego, CA, 596 USA). Data sets were either tested with a one-way ANOVA or a Student's t test. A value of $597 \mathrm{p}<0.05$ was considered significant.

\section{Data availability}

600 All data have been made available within this manuscript. Transgenic mosquito lines as well 601 as plasmids are available upon request. 


\section{Ethics statement}

604 Experiments were carried out in conformity with the 2010/63/EU directive of the European

605 Parliament on the protection of animals used for scientific purposes. Our animal care facility

606 received agreement \#I-67-482-2 from the veterinary services of the département du Bas Rhin

607 (Direction Départementale de la Protection des Populations). The use of animals for this

608 project was authorized by the French ministry of higher education, research and innovation

609 under the number APAFIS\#20562- 2019050313288887 v3. The generation and use of 610 transgenic lines (bacteria, mosquitoes, parasite) was authorized by the French ministry of 611 higher education, research and innovation under the number 3243.

\section{Funding}

614 This work was supported by the Laboratoire d'Excellence (LabEx) ParaFrap (grant LabEx 615 ParaFrap ANR-11-LABX-0024), by the Equipement d'Excellence (EquipEx) I2MC (grant 616 ANR-11-EQPX-0022), by the ERC Starting Grant N²60918, and by funding from CNRS, 617 Inserm, and the University of Strasbourg. DK is funded by a DFG postdoctoral fellowship $618($ KL 3251/1-1).

\section{Acknowledgements}

621 We thank Amandine Gautier for help with rearing mosquitoes and technical assistance during

622 infections with $P$. berghei, Sarra Manai for help with experiments, Jean-Daniel Fauny for 623 assistance during microscopy and the whole mosquito immune responses (MIR) team for 624 assistance with mosquito breeding and pupae collection.

625

\section{Authors contributions}

627 DK designed and performed experiments, analyzed data, made figures and wrote the draft.

628 KA cloned constructs, performed initial experiments and analyzed data. EM performed transgenesis of mosquitoes. SB edited the draft and helped with conceptualization of experiments. All authors edited and approved the manuscript for publication.

\section{Competing interests}

633 The authors declare no competing interests. 


\section{References}

Adolfi, A. et al. (2018) 'Multi-tissue GAL4-mediated gene expression in all Anopheles gambiae life stages using an endogenous polyubiquitin promoter', Insect Biochemistry and Molecular Biology. doi: 10.1016/j.ibmb.2018.03.005.

Bae, S., Park, J. and Kim, J. S. (2014) 'Cas-OFFinder: A fast and versatile algorithm that searches for potential off-target sites of Cas9 RNA-guided endonucleases', Bioinformatics. doi: 10.1093/bioinformatics/btu048.

Berghammer, A. J., Klingler, M. and Wimmer, E. A. (1999) 'A universal marker for transgenic insects', Nature. doi: 10.1038/46463.

Bhatt, S. et al. (2015) 'The effect of malaria control on Plasmodium falciparum in Africa between 2000 and 2015', Nature. doi: 10.1038/nature15535.

Douglas, R. G. et al. (2015) 'Active migration and passive transport of malaria parasites', Trends in Parasitology, pp. 357-362. doi: 10.1016/j.pt.2015.04.010.

Dragovic, S. M. et al. (2018) 'Immunization with AgTRIO, a Protein in Anopheles Saliva, Contributes to Protection against Plasmodium Infection in Mice', Cell Host and Microbe. doi: 10.1016/j.chom.2018.03.008.

Engler, C. and Marillonnet, S. (2014) 'Golden Gate cloning', Methods in Molecular Biology. doi: 10.1007/978-1-62703-764-8_9.

Francischetti, I. M. B. et al. (2002) 'Toward a catalog for the transcripts and proteins (sialome) from the salivary gland of the malaria vector Anopheles gambiae', Journal of Experimental Biology.

Fuchs, S., Nolan, T. and Crisanti, A. (2013) 'Mosquito transgenic technologies to reduce plasmodium transmission', Methods in Molecular Biology, 923, pp. 601-622. doi: 10.1007/978-1-62703-26-7_41.

Ghosh, A. K. and Jacobs-Lorena, M. (2009) 'Plasmodium sporozoite invasion of the mosquito salivary gland', Curr Opin Microbiol, 12. doi: 10.1016/j.mib.2009.06.010.

Gutscher, M. et al. (2008) 'Real-time imaging of the intracellular glutathione redox potential', Nature Methods. doi: 10.1038/nmeth.1212. 
Hayashi, H. et al. (2012) 'Anopheline anti-platelet protein from a malaria vector mosquito has anti-thrombotic effects in vivo without compromising hemostasis', Thrombosis Research. doi: 10.1016/j.thromres.2011.09.015.

Hillyer, J. F., Barreau, C. and Vernick, K. D. (2007) 'Efficiency of salivary gland invasion by malaria sporozoites is controlled by rapid sporozoite destruction in the mosquito haemocoel', International Journal for Parasitology. doi: 10.1016/j.ijpara.2006.12.007.

Hunt, T. M. and Bridges, C. B. (1916) Sex Linked Inheritance in Drosophila, Carnegie Institution of Washington.

Islam, A. et al. (2019) 'Anopheline antiplatelet protein from mosquito saliva regulates blood feeding behavior', Scientific Reports. doi: 10.1038/s41598-01939960-2.

Klug, D. and Frischknecht, F. (2017) 'Motility precedes egress of malaria parasites from oocysts', eLife, 6 .

Lee, J. H. et al. (1996) 'Sequential amplification of cloned DNA as tandem multimers using class- IIS restriction enzymes', Genetic Analysis Biomolecular Engineering. doi: 10.1016/S1050-3862(96)00164-7.

Manzoni, G. et al. (2015) 'A rapid and robust selection procedure for generating drug-selectable marker-free recombinant malaria parasites', Scientific Reports, 4(1), p. 4760. doi: 10.1038/srep04760.

Mayne, B. (1922) 'How Long Does a Mosquito Retain Malaria Parasites?', Public Health Reports (1896-1970). doi: 10.2307/4576379.

Mori, T., Hirai, M. and Mita, T. (2019) 'See-through observation of malaria parasite behaviors in the mosquito vector', Scientific Reports. doi: 10.1038/s41598-019-38529-3.

Natarajan, R. et al. (2001) 'Fluorescent Plasmodium berghei sporozoites and pre-erythrocytic stages: A new tool to study mosquito and mammalian host interactions with malaria parasites', Cellular Microbiology, 3(6), pp. 371-379. doi: 10.1046/j.1462-5822.2001.00117.x.

De Niz, M. et al. (2019) '3D imaging of undissected optically cleared Anopheles stephensi mosquitoes infected with Plasmodium parasites', bioRxiv. doi: $10.1101 / 682054$. 
De Niz, M. et al. (2019) 'Intravital imaging of host-parasite interactions in skin and adipose tissues', Cellular Microbiology. doi: 10.1111/cmi.13023.

O'Brochta, D. A. et al. (2019) 'Is Saglin a mosquito salivary gland receptor for Plasmodium falciparum?', Malaria Journal. doi: 10.1186/s12936-018-2634-5.

Osanai-Futahashi, M. et al. (2012) 'A visible dominant marker for insect transgenesis', Nature Communications. doi: 10.1038/ncomms2312.

Pondeville, E. et al. (2014) 'Efficient ФC31 integrase-mediated site-specific germline transformation of Anopheles gambiae', Nature Protocols, 9(7), pp. 1698-1712. doi: 10.1038/nprot.2014.117.

Ribeiro, J. M. C. and Francischetti, I. M. B. (2003) 'Role of Arthropod Saliva in

Schindelin, J. et al. (2012) 'Fiji: an open-source platform for biological-image

Soille, P. and Vincent, L. M. (1990) '<title $>$ Determining watersheds in digital pictures via flooding simulations</title>', in Visual Communications and Image Processing '90: Fifth in a Series. doi: 10.1117/12.24211.

Sturm, A. et al. (2006) 'Manipulation of host hepatocytes by the malaria parasite for delivery into liver sinusoids.', Science (New York, N.Y.), 313(5791), pp. 1287-1290. doi: 10.1126/science.1129720.

744

TerMaat, J. R. et al. (2009) 'Gene synthesis by integrated polymerase chain assembly and PCR amplification using a high-speed thermocycler', Journal of Microbiological Methods. doi: 10.1016/j.mimet.2009.09.015. Plasmodium Invasion of the Mosquito Midgut ', mSphere. doi: 10.1128/msphere.00692-20.

True, J. R. et al. (2005) 'Drosophila tan encodes a novel hydrolase required in pigmentation and vision.', PLoS genetics, 1(5). doi: 10.1371/journal.pgen.0010063.

Volohonsky, G. et al. (2015) 'Tools for Anopheles gambiae Transgenesis', G3 (Bethesda); Genes|Genomes|Genetics, 5(6), pp. 1151-1163. doi: 10.1534/g3.115.016808. 
760 Wang, J. et al. (2013) 'Anopheles gambiae circumsporozoite protein--binding 761 protein facilitates Plasmodium infection of mosquito salivary glands', $J$ Infect 762 Dis, 208. doi: 10.1093/infdis/jit284.

764 Wang, L. T. et al. (2020) 'A Potent Anti-Malarial Human Monoclonal Antibody 765 Targets Circumsporozoite Protein Minor Repeats and Neutralizes Sporozoites in 766 the Liver', Immunity. doi: 10.1016/j.immuni.2020.08.014.

767

768 Wells, M. B. and Andrew, D. J. (2019) 'Anopheles salivary gland architecture 769 shapes plasmodium sporozoite availability for transmission', mBio. doi:

$770 \quad 10.1128 / \mathrm{mBio} .01238-19$.

Wittkopp, P. J., True, J. R. and Carroll, S. B. (2002) 'Reciprocal functions of the Drosophila Yellow and Ebony proteins in the development and evolution of

774 pigment patterns', Development.

776 Yoshida, S. et al. (2008) 'Inhibition of collagen-induced platelet aggregation by 777 anopheline antiplatelet protein, a saliva protein from a malaria vector mosquito', 778 Blood. doi: 10.1182/blood-2007-06-097824.

780 Yoshida, S. and Watanabe, H. (2006) 'Robust salivary gland-specific transgene expression in Anopheles stephensi mosquito', Insect Molecular Biology. doi: 10.1111/j.1365-2583.2006.00645.x. 

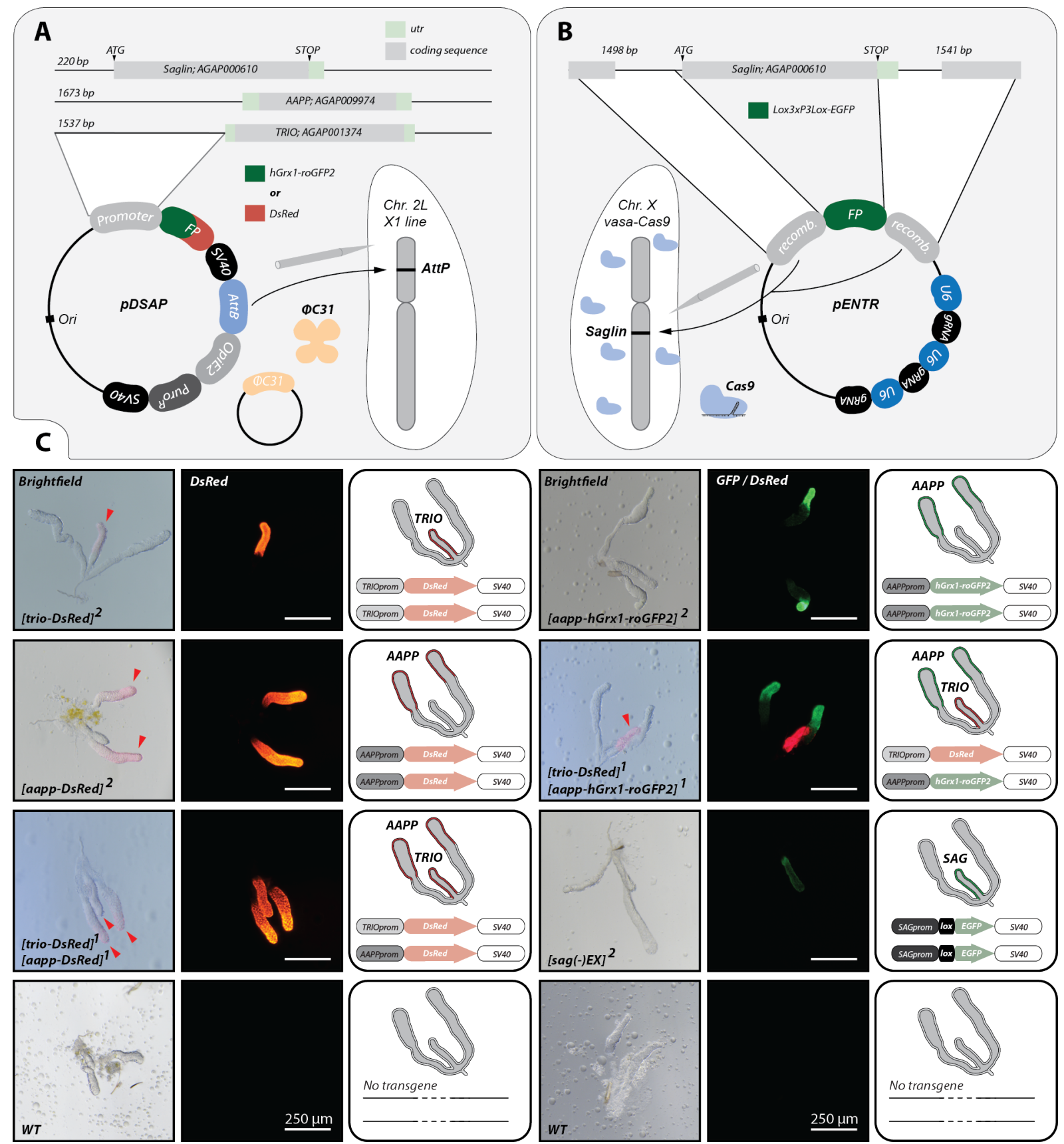

Figure 1. Lobe-specific reporter expression observed in female aapp-DsRed, aapp-hGrx1roGFP2, trio-DsRed and sag(-)EX mosquitoes.

A) ФC31 integrase-mediated transgene insertion. The aapp, trio and saglin promoter sequences were fused to DsRed or hGrx1-roGFP2 within the pDSAP vector which enables chemical selection of mosquito larvae by puromycin. Transgenes were integrated by ФC31 integrase-mediated recombination into the $\mathrm{X} 1$ line harbouring an attP site on chromosome $2 \mathrm{~L}$. B) Cas9-mediated knockin of a fluorescence cassette in the saglin gene (AGAP000610). Upstream and downstream sequences of saglin were cloned into the pENTR vector flanking a fluorescence cassette expressing EGFP under the control of the $3 x P 3$ promoter. Three guides targeting saglin controlled by U6 promoters were encoded on the same pENTR vector. Transgenesis was performed in mosquitoes with germline-specific expression of Cas9 (vasaCas9). C) Fluorescent reporter expression in trio-DsRed, aapp-DsRed, aapp-hGrx1-roGFP2 and $\operatorname{sag}(-) E X$ expressing mosquito females in comparison to wild type (G3). Representative 
799 images of salivary glands are shown in brightfield beside an image of the same gland in the 800 relevant fluorescence channel. Red arrows in brightfield images highlight the pink parts of 801 aapp-DsRed and trio-DsRed salivary glands in the absence of fluorescence excitation 802 indicating the high expression of DsRed. The illustration on the right of each panel indicates 803 the genotype of the gland as well as the observed fluorescence pattern. Scale bars: $250 \mu \mathrm{m}$. 
A
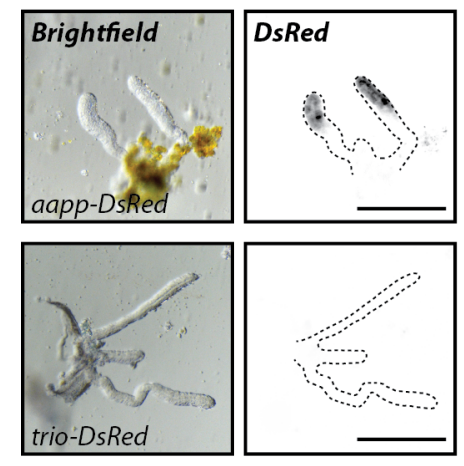

B

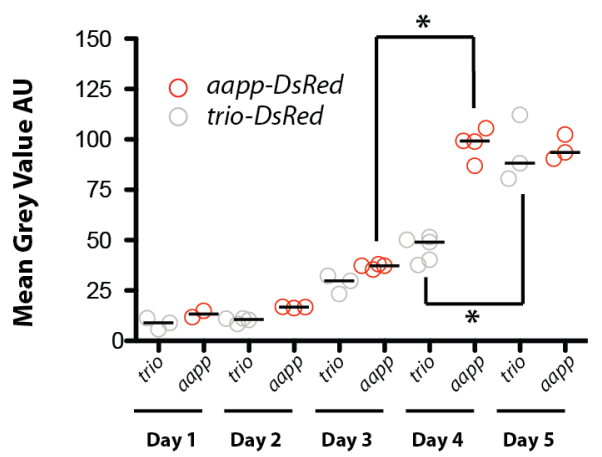

C $\quad n=\begin{array}{llll}62 & 59 & 39 & 36\end{array}$

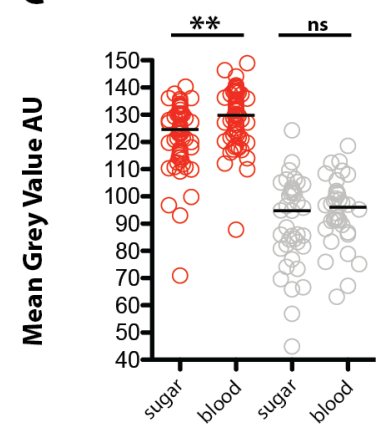

Figure 2. Onset, strength and inducibility by blood feeding of reporter expression in aapp-DsRed and trio-DsRed mosquitoes.

809 A) Images of salivary glands dissected directly after mosquitoes emerged. DsRed expression was observed in aapp-DsRed but not in trio-DsRed expressing salivary glands. Scale bar: 250 $\mu \mathrm{m}$. B) Mean fluorescence intensity measurements of dissected aapp-DsRed and trio-DsRed salivary glands from day one to day five after hatching. Data were normalized for the highest exposure time. Each dot represents a single gland (2-5 glands / timepoint) and the bars, mean values. ${ }^{*} \mathrm{p}<0.05$, Mann Whitney test. C) The blood meal inducibility of the trio and the aapp promoters was assessed by providing sibling females either with a blood meal or sugar solution. Approximately $24 \mathrm{~h}$ later, salivary glands were dissected, imaged and the mean DsRed fluorescence intensity was quantified. Data pooled from three independent experiments. Same representation and statistical analysis as in $(B),{ }^{*} p=0.0015$. 

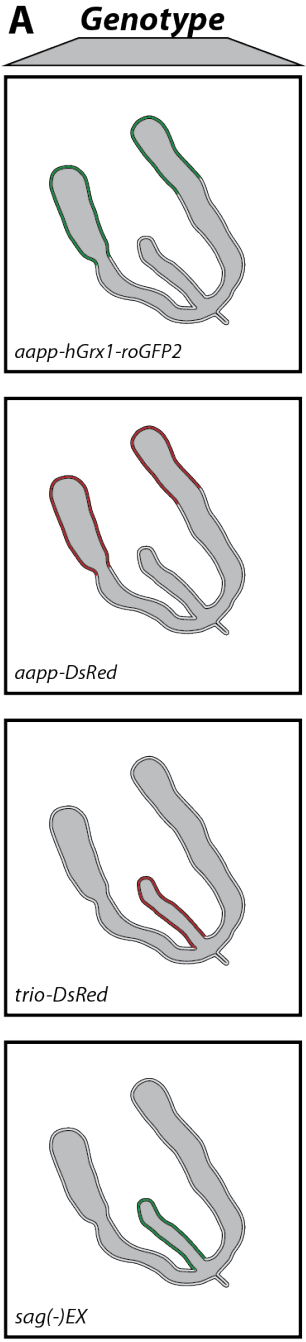

B

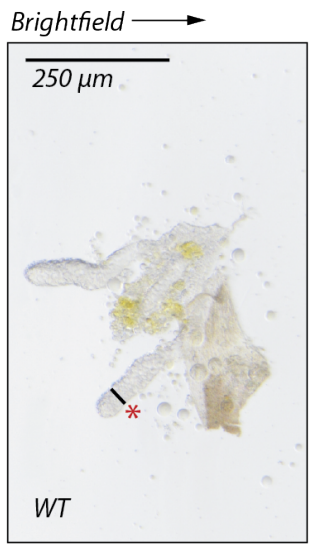

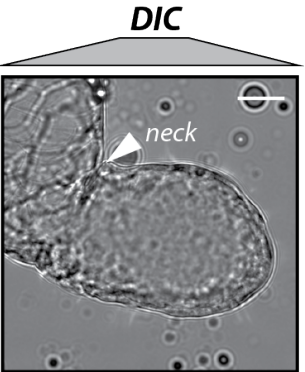
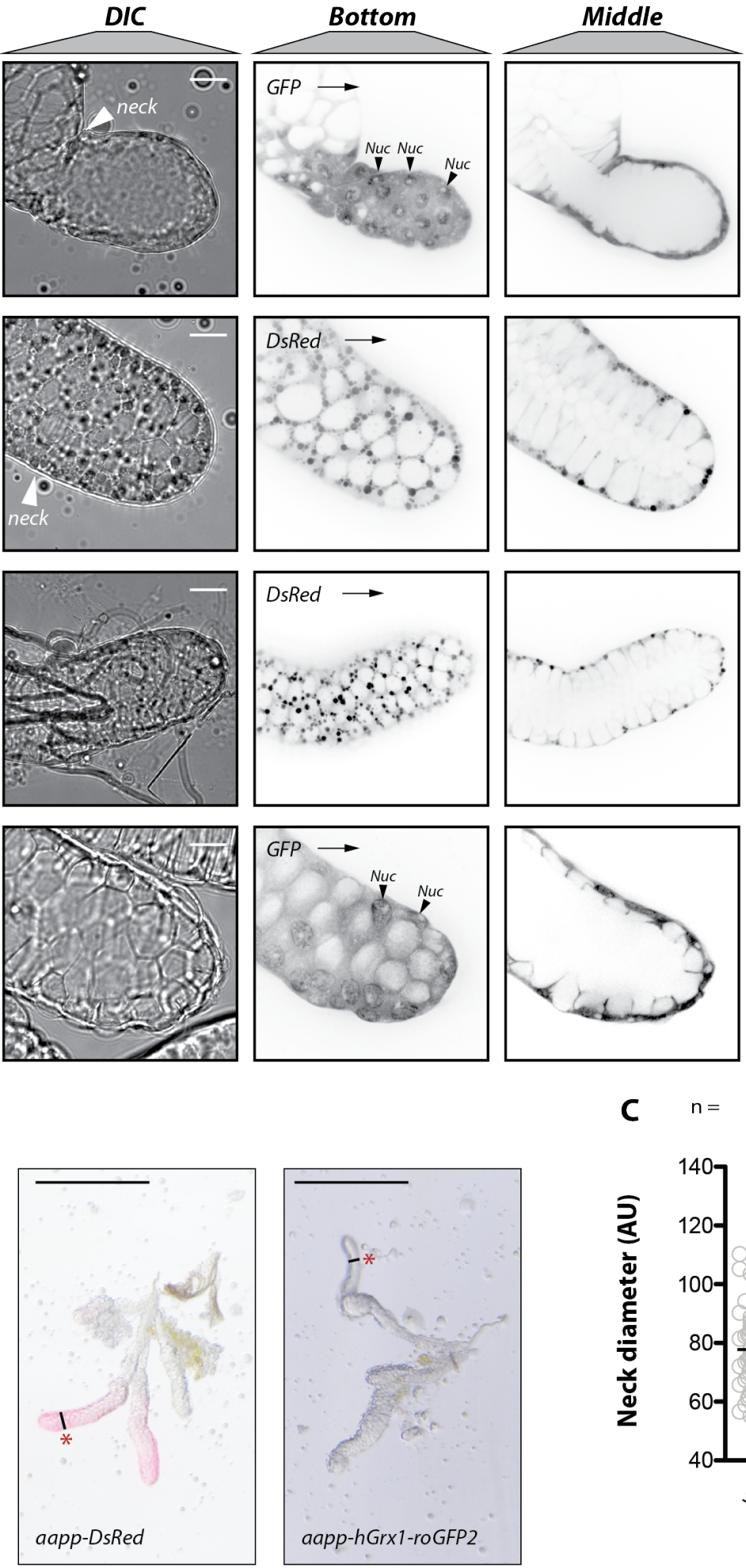
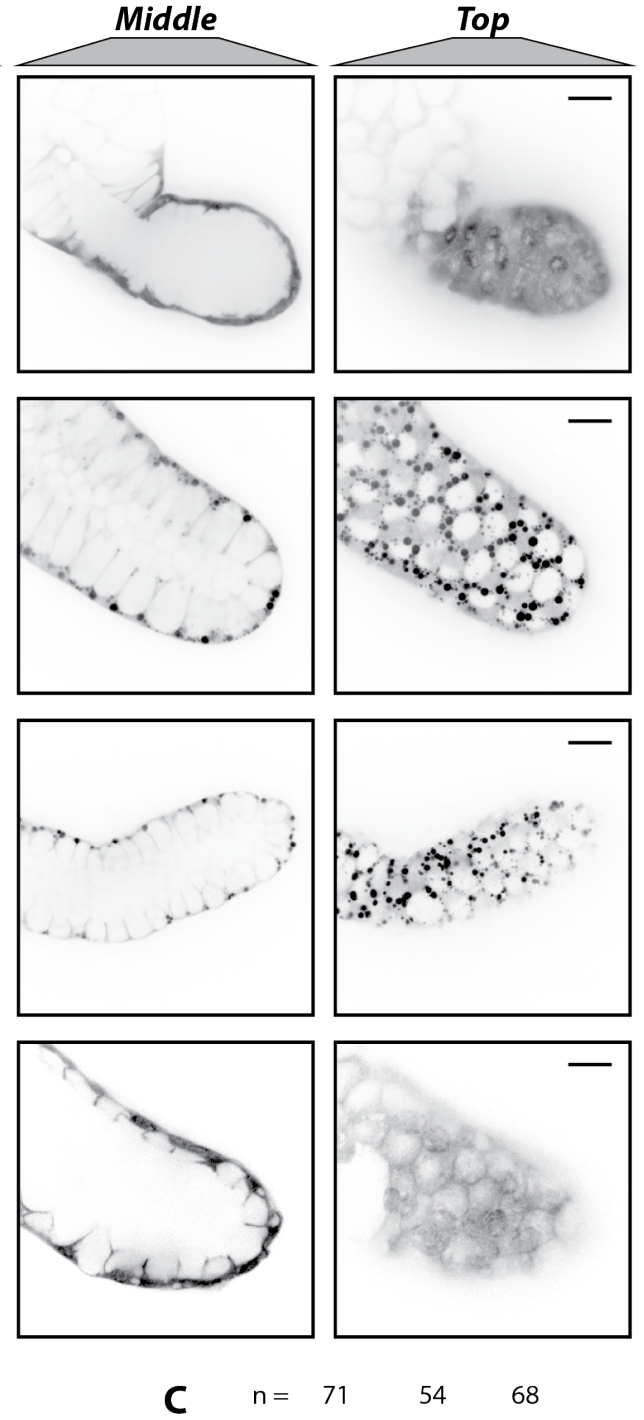

C $\quad \mathrm{n}=\begin{array}{llll}71 & 54 & 68\end{array}$

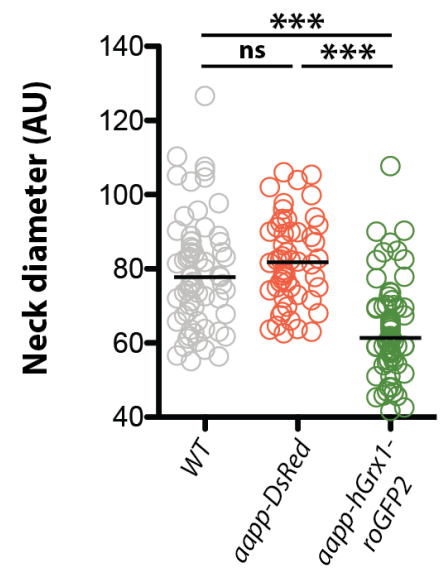

Figure 3. Different subcellular localizations of the expressed DsRed, EGFP and hGrx1roGFP2 fluorescent reporters.

A) Differential interference contrast (DIC) and confocal fluorescence images of distal (aappDsRed, trio-DsRed) or middle (trio-DsRed, sag(-)EX) salivary gland lobes. Different zsections (top, middle and bottom) of the same glands are displayed in the relevant fluorescence channel. The ,neck“ regions of the aapp-DsRed and aapp-hGrxl-roGFP2 expressing lobes are indicated by white arrows. Note that DIC and fluorescence images were 
829 taken with a Hamamatsu Orca Flash 4.0 V1 and a Yokogawa CSU X-1 camera, respectively.

830 Images between different channels are therefore not completely matching although depicting

831 the same salivary gland. The illustration on the left depicts the genotype and corresponding 832 expression pattern. Scale bars: $20 \mu \mathrm{m}$. B) Brightfield images of female wild type (G3), aapp833 DsRed and aapp-hGrxl-roGFP2 salivary glands. The ,neck“ is indicated by a red asterisk 834 and the measured diameter by a black line. Scale bars: $250 \mu \mathrm{m}$. C) Diameter measurements of 835 the distal lobe neck region of aapp-DsRed and aapp-hGrx1-roGFP2 in comparison to wild 836 type (G3). The number of measurements is indicated above each genotype. Note that only the 837 diameter of one distal lobe per gland was measured. Data represent $\geq 3$ independent 838 experiments. ${ }^{* * *} \mathrm{p}<0.0001$, one-way-ANOVA (Bonferroni's Multiple Comparison Test). 

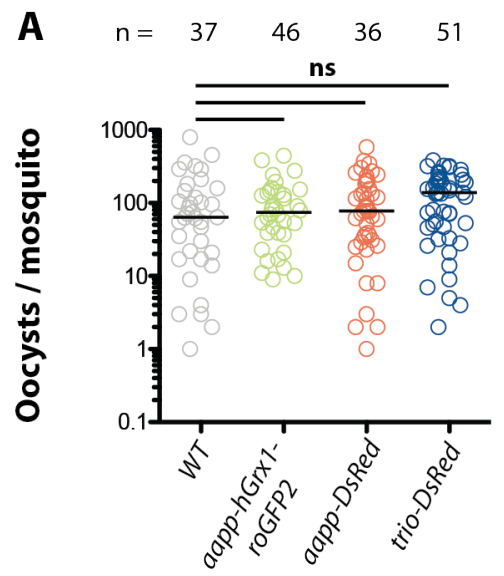

B

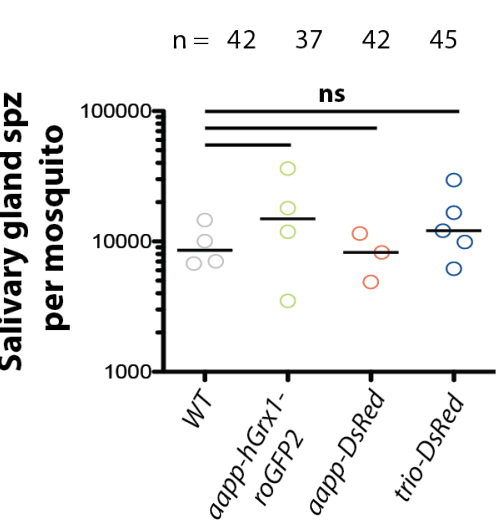

C

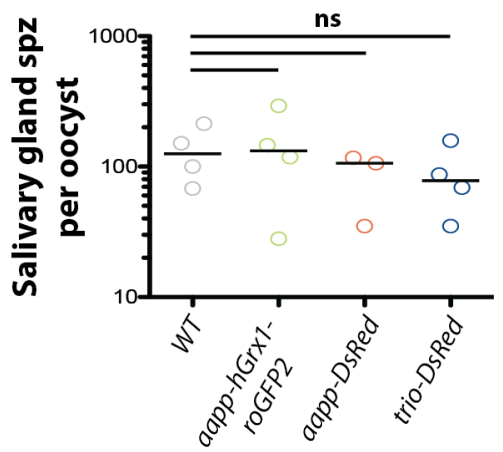

842

843

844

845

846

847

848

849

850

851

852

Figure 4. Oocyst densities and salivary gland colonization in aapp-DsRed, aapp-hGrx1roGFP2 and trio-DsRed mosquitoes infected with $P$. berghei.

Oocyst (A) and salivary gland sporozoite (spz) (B) counts in infected aapp-DsRed, aapphGrx1-roGFP2 and trio-DsRed females in comparison to wild type (G3) females. Data pooled from three biological replicates. The total number of dissected mosquitoes is given above each genotype. C) Number of salivary gland sporozoites (spz) per oocyst for all three reporter lines in comparison to wild type (WT: G3). The invasion rate was calculated using the data shown in (A) and (B). Each dot represents a single mosquito (A) or the result of a counting (B and $\mathrm{C}$ ), the median is indicated by a bar. All data were tested for significance using a oneway-ANOVA (Kruskal-Wallis test). ns: not significant ( $\mathrm{p}>0.05)$. 

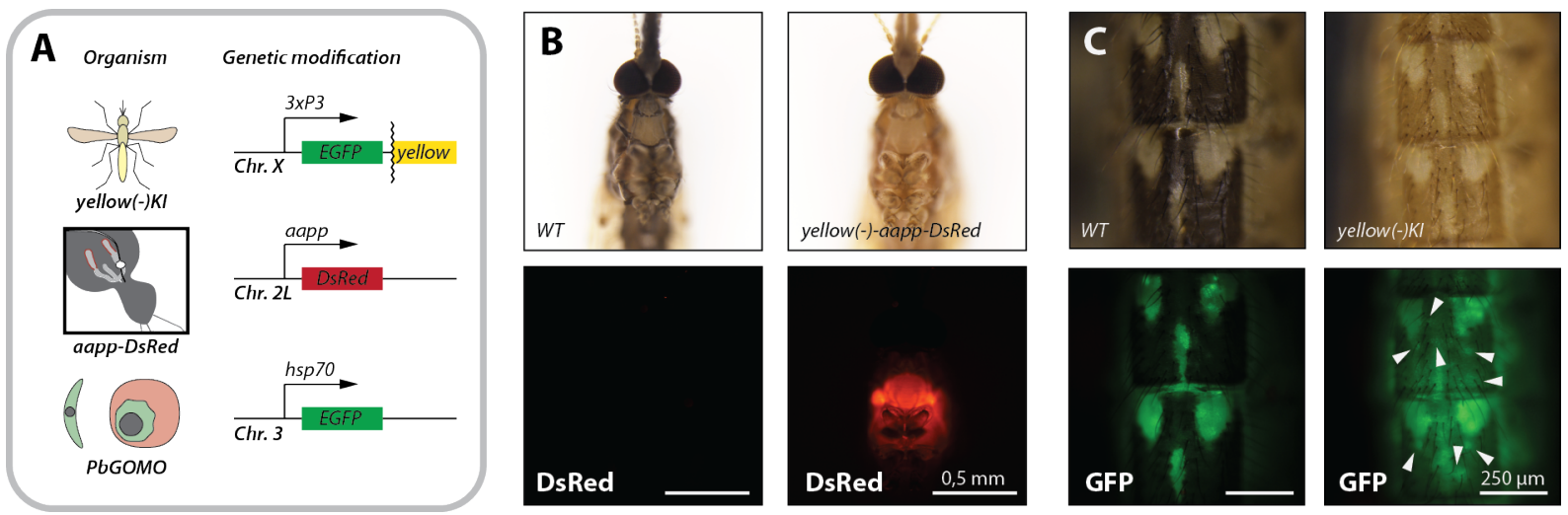

Figure 5. Salivary gland reporter lines as tool for in vivo imaging.

A) Genetic models used for in vivo imaging. Mosquitoes carrying the aapp-DsRed transgene were crossed with yellow(-)KI mosquitoes displaying reduced black pigmentation. Infections were carried out using the highly fluorescent $P$. berghei line $\Delta p 230 p-G F P$ constitutively expressing EGFP (Manzoni et al., 2015). B) Pigmentation phenotype and salivary glandspecific DsRed expression of yellow(-)-aapp-DsRed mosquitoes in comparison to wild type (WT: Ngousso). Brightfield and DsRed expression of the same field of view. Scale bar: 0.5 mm. C) Images of the abdomen of infected yellow(-)KI and wild type (Ngousso) mosquitoes. Brightfield and GFP expression of the same field of view. White arrows point towards oocysts visible through strong pigmented areas of the cuticle of yellow(-)KI mosquitoes. Scale 

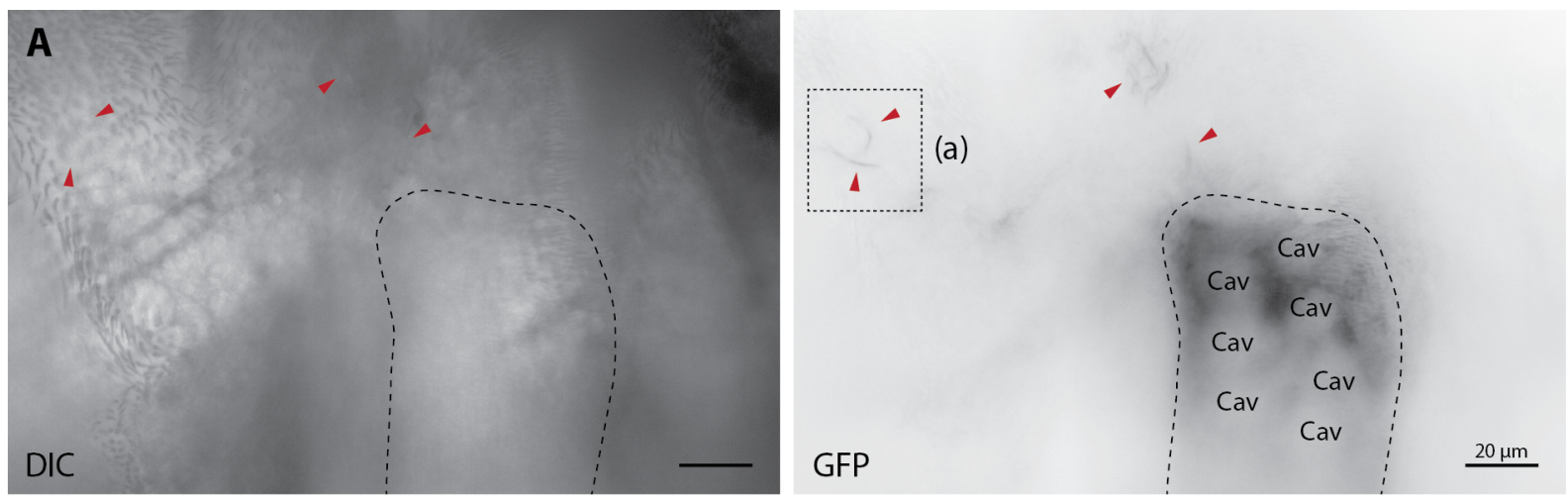

B

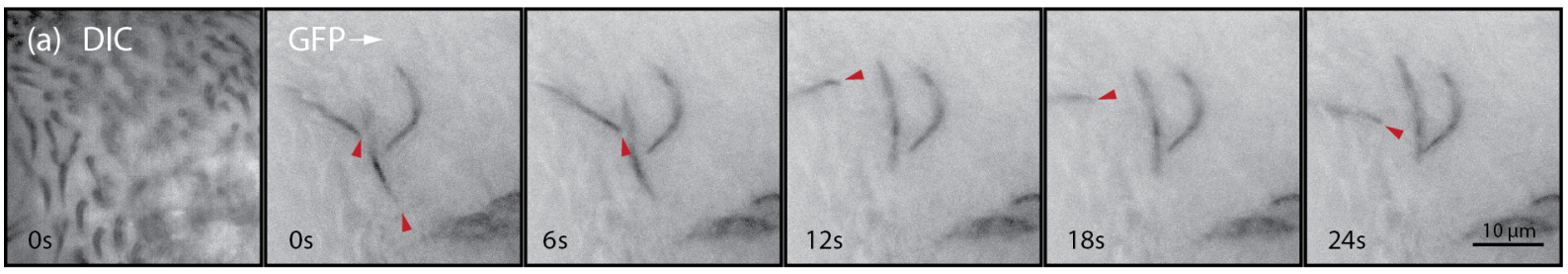

C

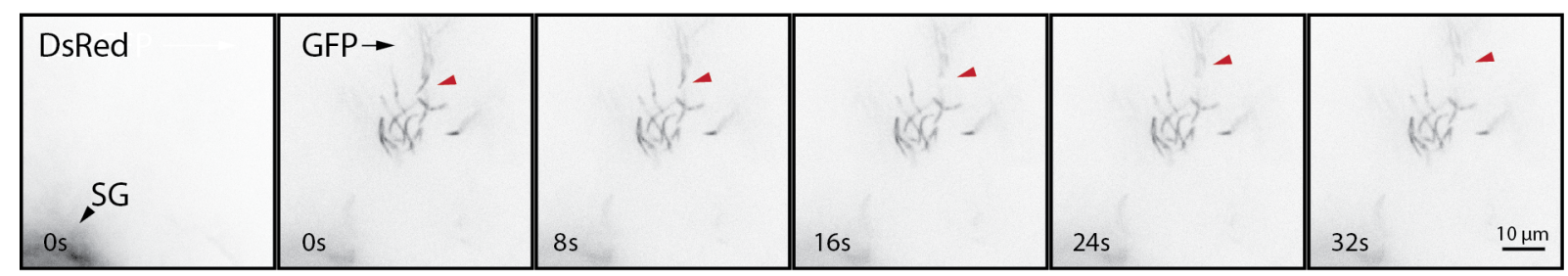

D

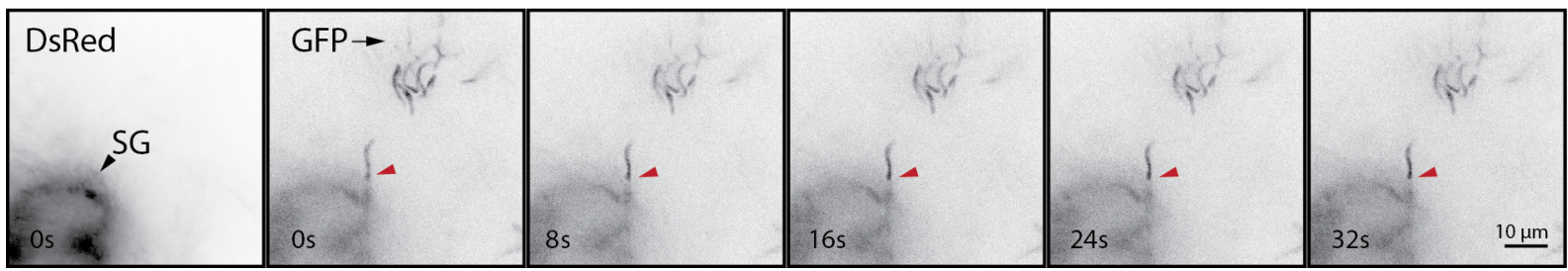

Figure 6. Confocal imaging of sporozoite salivary gland interactions in live mosquitoes.

A) Thorax view of a yellow(-)-aapp-DsRed female mosquito infected with $P$. berghei $\triangle p 230 p-G F P$. Differential interference contrast (DIC) image on the left and the GFP signal on the right. Note that the DsRed expression in the salivary glands is strong enough to be visible in the GFP channel. Red arrows point towards sporozoites and the dashed line in the DIC image indicates the position of the salivary gland as seen in the GFP channel. Scale bar: 20 $\mu \mathrm{m}$. B) Zoom on a group of sporozoites sequestered in close proximity to the salivary gland in the area boxed as (a) in the previous image (A). DIC image and a time series of the GFP signal with $6 \mathrm{~s}$ between frames. Red arrows point towards sporozoites displaying movement. Scale bar: $10 \mu \mathrm{m}$. C,D) Accumulation of sporozoites close to the salivary gland. $\mathbf{C}$ and $\mathbf{D}$ show the same salivary gland with altered focus and shifted field of view. DsRed signal (left) to indicate the position of the salivary gland (SG), and time series of the GFP signal with $8 \mathrm{~s}$ between frames. The red arrow points towards sporozoites displaying active movement (C) or towards a sporozoite in contact with the salivary gland (D). Scale bar: $10 \mu \mathrm{m}$. Note that imaging was performed with a Hamamatsu Orca Flash 4.0 V1 camera for A) and B) or a Yokogawa CSU X-1 for C) and D) using a 63-fold magnification objective (N.A. 1.4). 

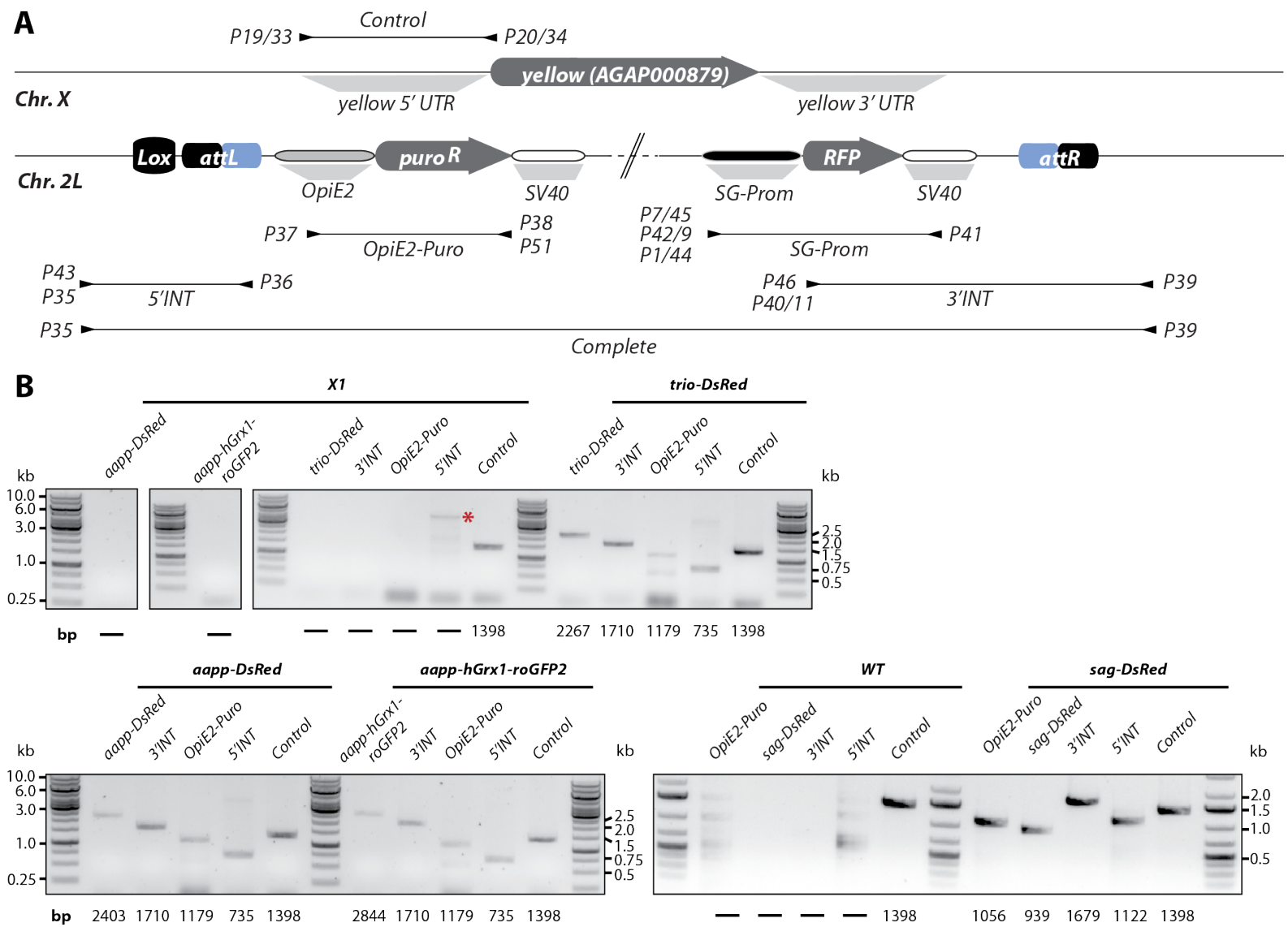

Figure S1. Genotyping of the salivary gland reporter lines aapp-DsRed, aapp-hGrx1roGFP2 and trio-DsRed.

A) Schematic representation of salivary gland reporter transgenesis cassettes inserted on chromosome 2L. Fusion of $a t t B$ and $a t t P$ sites generate $a t t L$ and attR sites after integration. The Lox site upstream of the transgene is a remnant of the fluorescence cassette initially required to select the docking line X1. Note that the illustration is not drawn to scale. B) Genotyping of generated transgenic mosquito colonies in comparison to wild type (WT: G3) or the parental line X1. Five different analytical PCRs were performed. The 5'INT and 3'INT PCRs amplify the integration borders upstream and downstream of the transgene, respectively. The presence of the puromycin resistance cassette was tested (OpiE2-Puro PCR). To ensure integration of the relevant fluorescence cassette, the PCRs aapp-DsRed, aapp-hGrx1-roGFP2, trio-DsRed and sag-DsRed were performed. In addition, a PCR control amplifying the 5'UTR of the yellow gene on chromosome X was included. Genotyping primers and amplified sequences are indicated with arrowheads and thin black lines in the scheme (A). The red asterisk marks an unspecific band only observed in the control X1. 
A
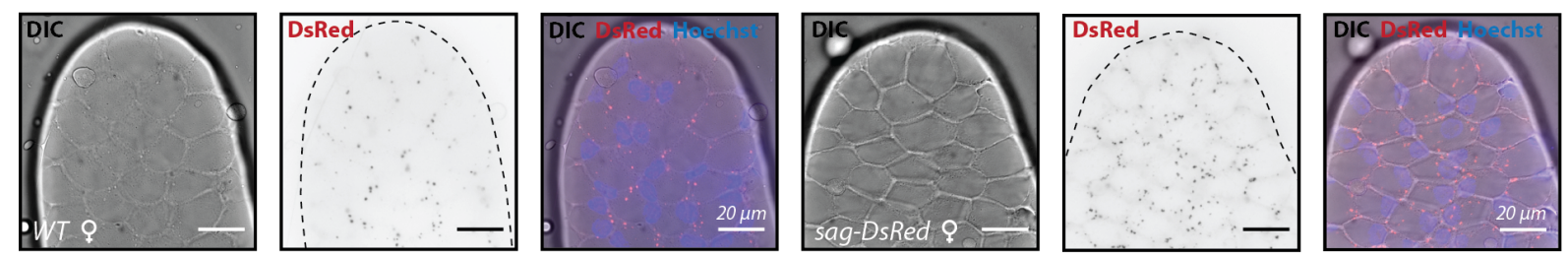

B

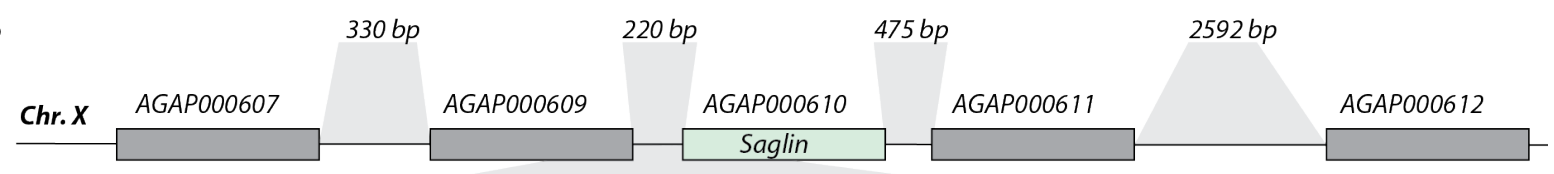

Figure S2. The intergenic sequence between AGAP000609 and AGAP000610 (saglin) displays no promoter activity.

TAACTTTCATGCGTACAATGTGTATGATCACTGATGACAAAGCGCTTGGGTGGGCGCTT GCTGCTGAAGCGTGGCTGACAAATGGGGGGGGGGCTTGAACAAATATTTGCACACACGAT AATGCTCTTGTTTGCCATGCGCTGACGGAAGACAAATCGCTGCAAGCGACGGCTCAAACC TGCTCAAACCAGGGAAGGTTTGCATAAGGTTCACTTATAAATG

A) Images of salivary glands from a sag-DsRed and a wild type female (Ngousso). Dissected glands were stained with Hoechst 33342 to detect the nuclei of acinar cells. The dotted black line in DsRed images indicates the outline of the salivary gland lobe observed in DIC. Scale bar: $20 \mu \mathrm{m}$. B) Genomic context of saglin (AGAP000610) with neighboring genes. The length of intergenic sequences is indicated above the scheme and the sequence tested for promoter activity located between AGAP000609 and saglin is shown. The stop codon TAA of AGAP000609 and the start codon of saglin are highlighted in black. The length of the cloned sequenced intergenic sequence was 220 bp (without stop codon of AGAP000609 and start codon of saglin). Note that the illustration is not drawn to scale. 


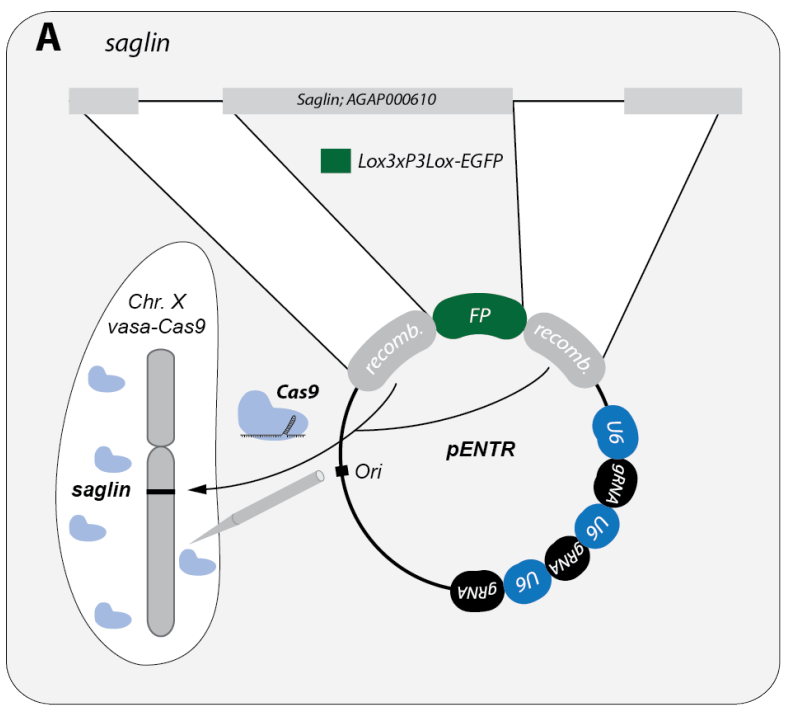

B yellow
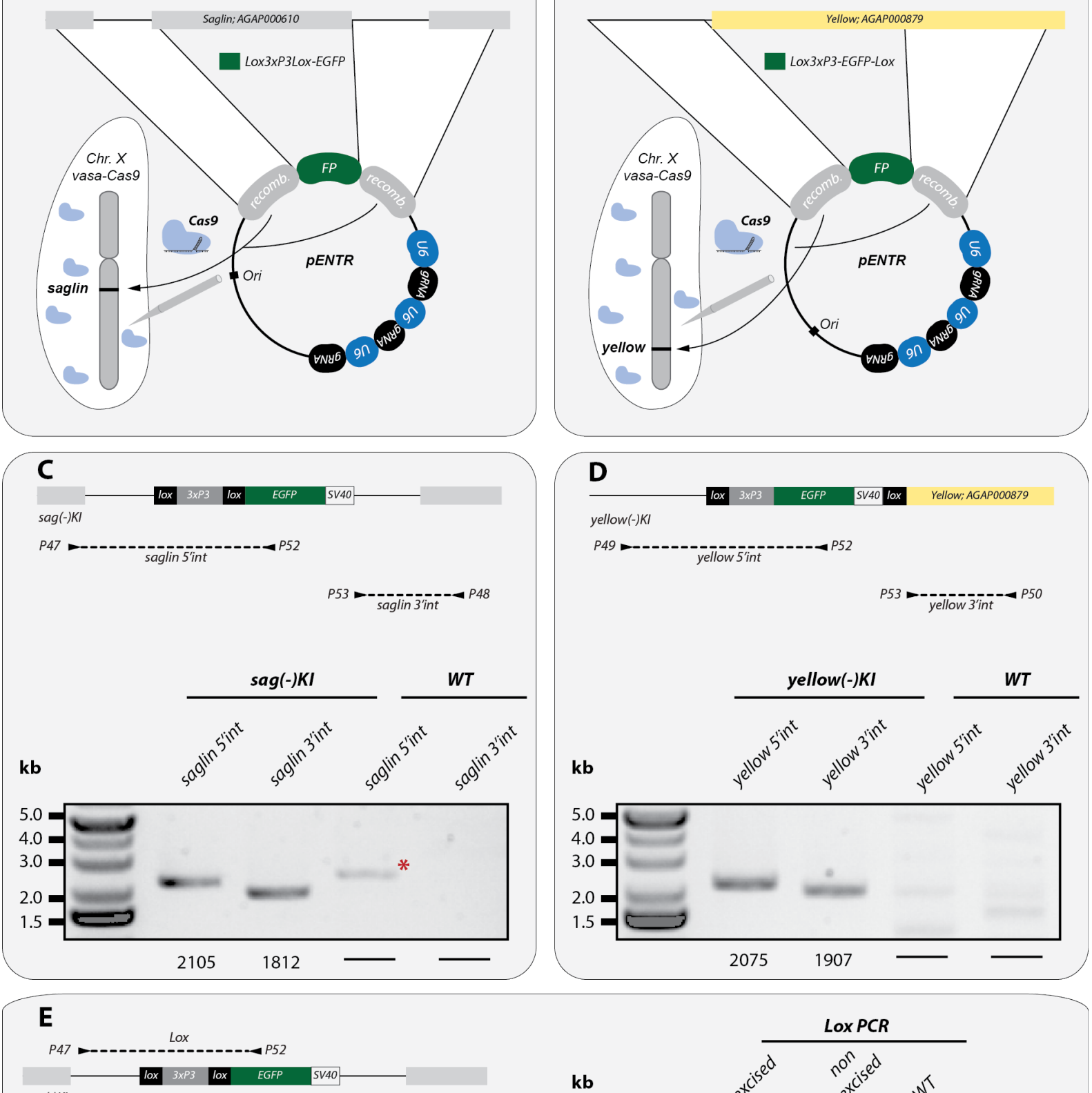

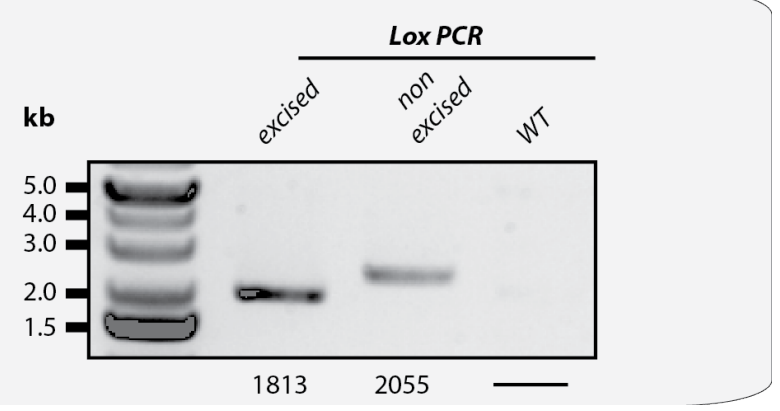

\section{Figure S3. Genotyping of sag(-)KI, sag(-)EX and yellow(-)KI mosquitoes.}

Sag(-)KI (A) and yellow(-)KI (B) mosquitoes were generated by injecting embryos expressing Cas9 (vasa-Cas9) with plasmids carrying repair templates that contain a Lox3xP3Lox-EGFP or a Lox3xP3-EGFP-Lox cassette flanked with upstream and downstream sequences of the respective targeted locus, in combination with three guide RNAs specific for the saglin (AGAP000610) or yellow gene (AGAP000879). Genotyping of the 5' and 3' integration borders revealed successful integration of the transgenes into saglin (C) and yellow (D). The red asterisk marks an unspecific PCR product observed in the wild type control (WT: Ngousso). E) To investigate the native expression of the saglin promoter, the $3 x P 3$ promoter 
929 initially used to select transgenic sag(-)KI mosquito larvae was removed by Cre-mediated 930 excision. The loss of the 3xP3Lox sequence in sag(-)EX mosquitoes was confirmed by PCR. 


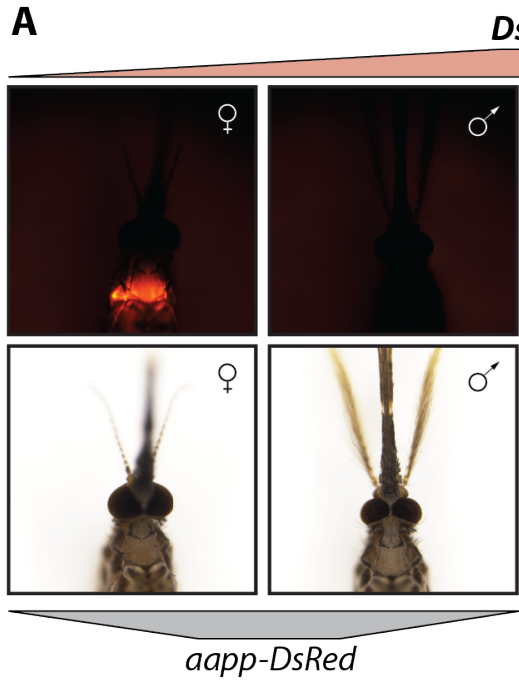

DsRed
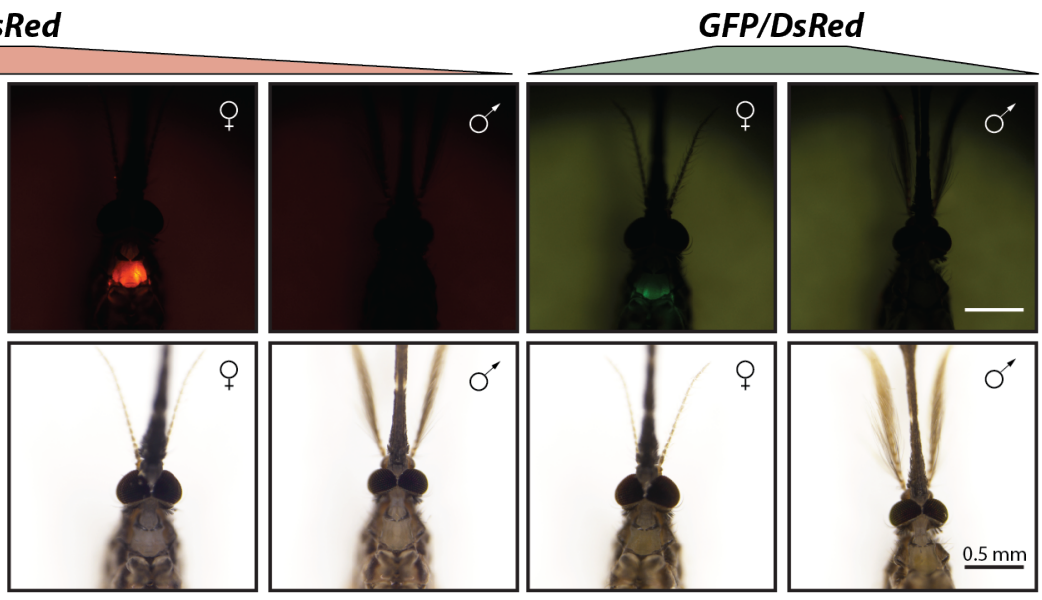

trio-DsRed

aapp-hGrx1-roGFP2

B

DsRed

GFP/DsRed

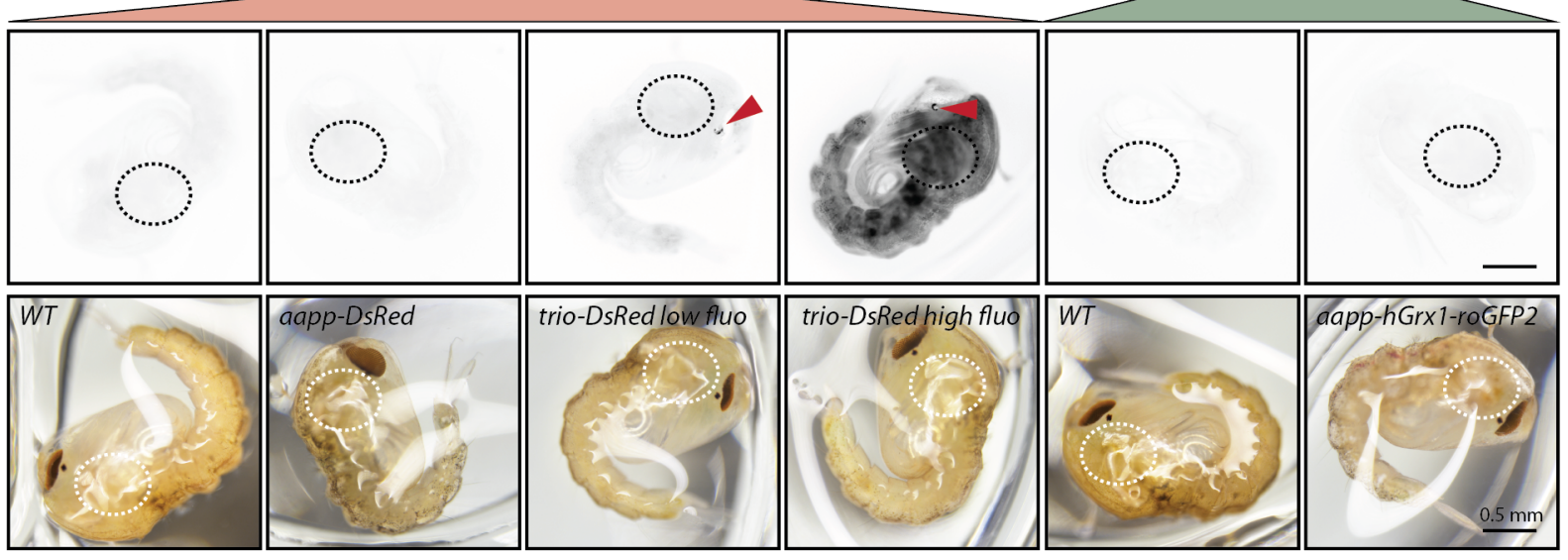

\section{Brightfield}

C

Age
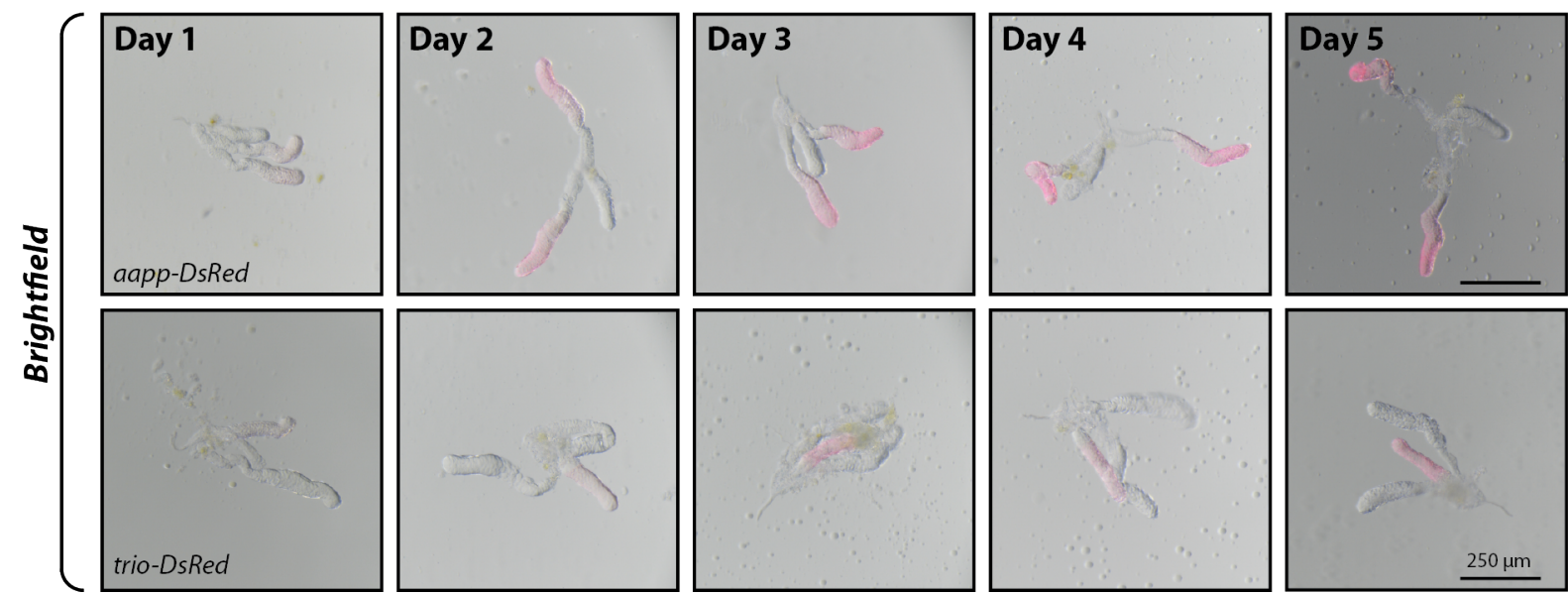

Figure S4. Expression driven by trio and aapp promoters is female- and adult-specific and the fluorescent reporter accumulates after the emergence of adults.

A) Fluorescence and brightfield images of female / male pairs of trio-DsRed, aapp-DsRed and aapp-hGrxl-roGFP2 mosquitoes. Note that all depicted mosquitoes were bred in synchrony and were seven day old (+/- 1 day). Scale bar: $0.5 \mathrm{~mm}$. B) Fluorescence and brightfield images of aapp-DsRed, trio-DsRed and aapp-hGrxl-roGFP2 pupae in comparison 
940 to wild type (G3). Dotted circles indicate the putative position of the salivary glands. Trio941 DsRed pupae with low and high DsRed body expression are shown for comparison. The red 942 arrows indicate DsRed positive ocelli observed in most trio-DsRed pupae. Scale bar: $0.5 \mathrm{~mm}$. 943 C) Brightfield images of aapp-DsRed and trio-DsRed female salivary glands from day 1 to 944 day 5 after hatching (a single image per day and per line). All images were acquired using the 945 same settings. Scale bar: $250 \mu \mathrm{m}$.

946 

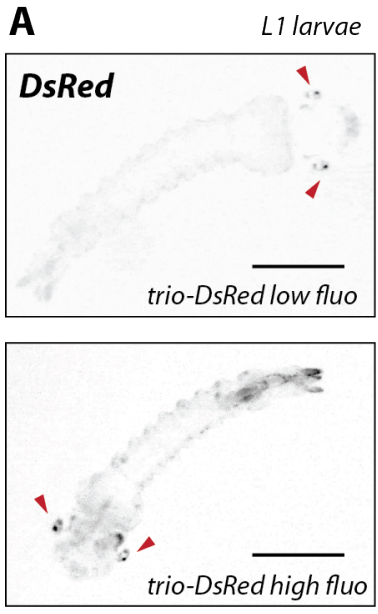

C
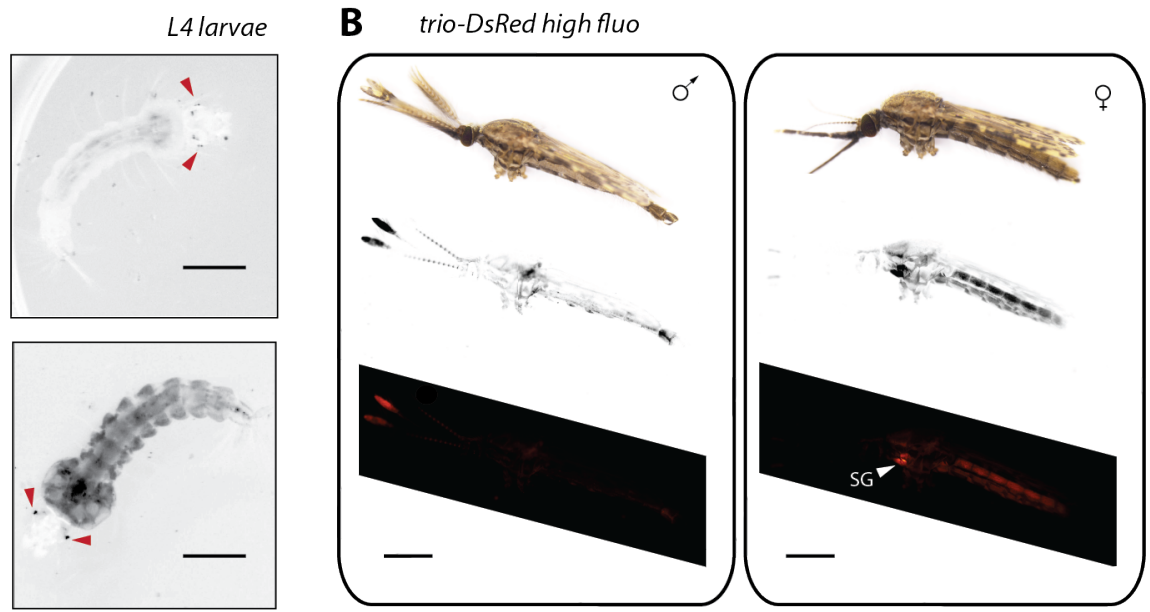

D
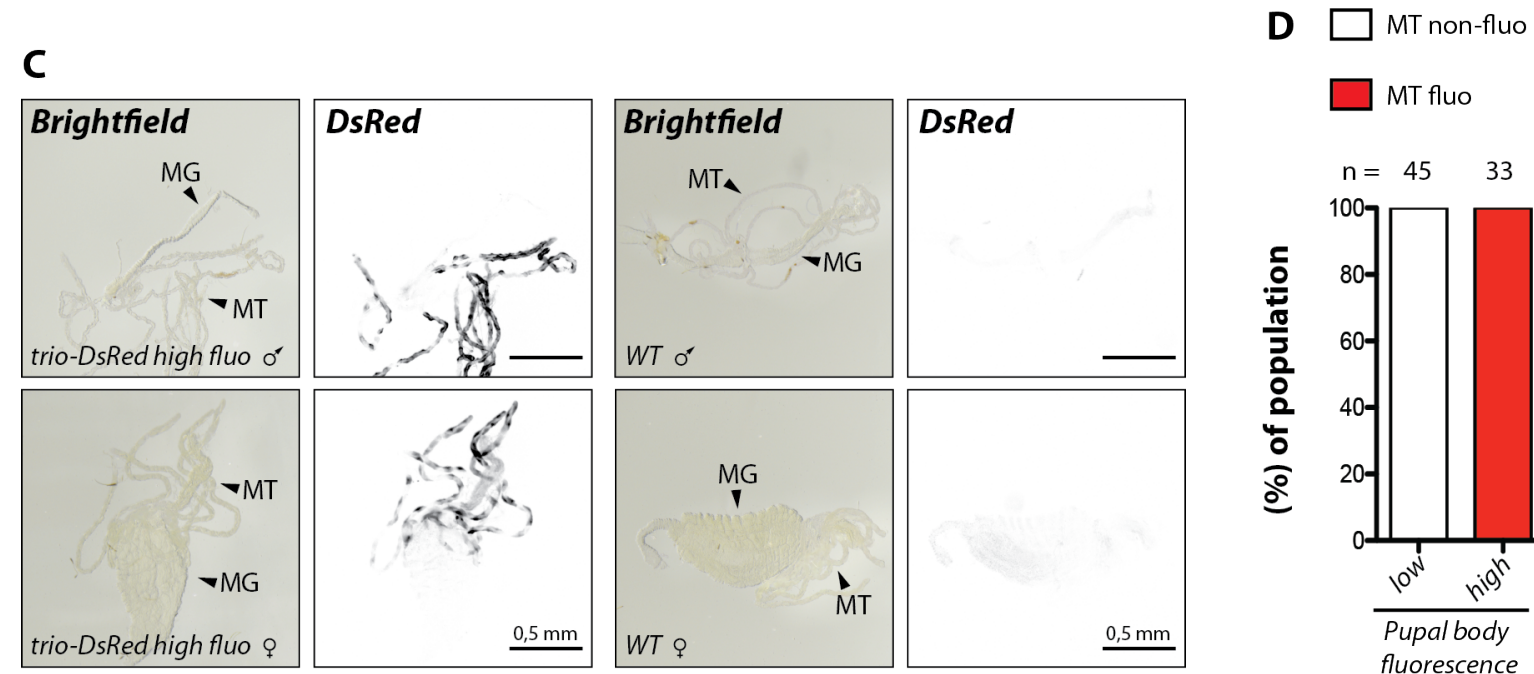

MT fluo

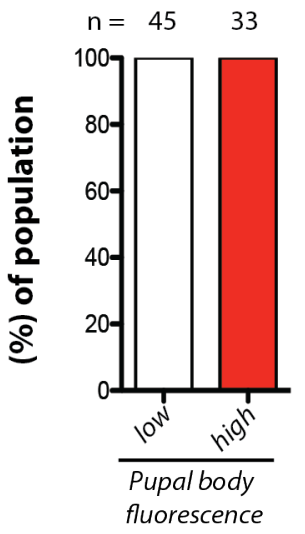

Figure S5. DsRed expression in Malphigian tubules (MT) of trio-DsRed mosquitoes is linked to ubiquitous DsRed expression in larvae, pupae and adults.

A) Trio-DsRed L1 and L4 larvae were classified as trio-DsRed low fluo or trio-DsRed high fluo according to their body DsRed fluorescence intensity. Red arrowheads point towards the DsRed positive eyes observed in the majority of larvae. Scale bar L1: $250 \mu \mathrm{m}$. Scale bar L4: $0.5 \mathrm{~mm}$. B) Whole body fluorescence of trio-DsRed high fluo male and female mosquitoes. From top to bottom: brightfield, inverted DsRed fluorescence signal displayed in black and white and in red on black. Note that images were stitched from two separate images showing either the head or the abdominal part. Scale bar: $0.5 \mathrm{~mm}$. C) Representative images of male and female midguts dissected from mosquitoes that displayed high body fluorescence at the pupal stage in comparison to wild type (WT: G3). Malphigian tubules (MT) and midguts (MG) are indicated by black arrows. Scale bar: $0.5 \mathrm{~mm}$. D) Correlation of high body fluorescence at the pupal stage with DsRed expression in malphigian tubules (MT). Data were collected from two different mosquito generations. The number of analyzed mosquitoes is indicated above each column. 

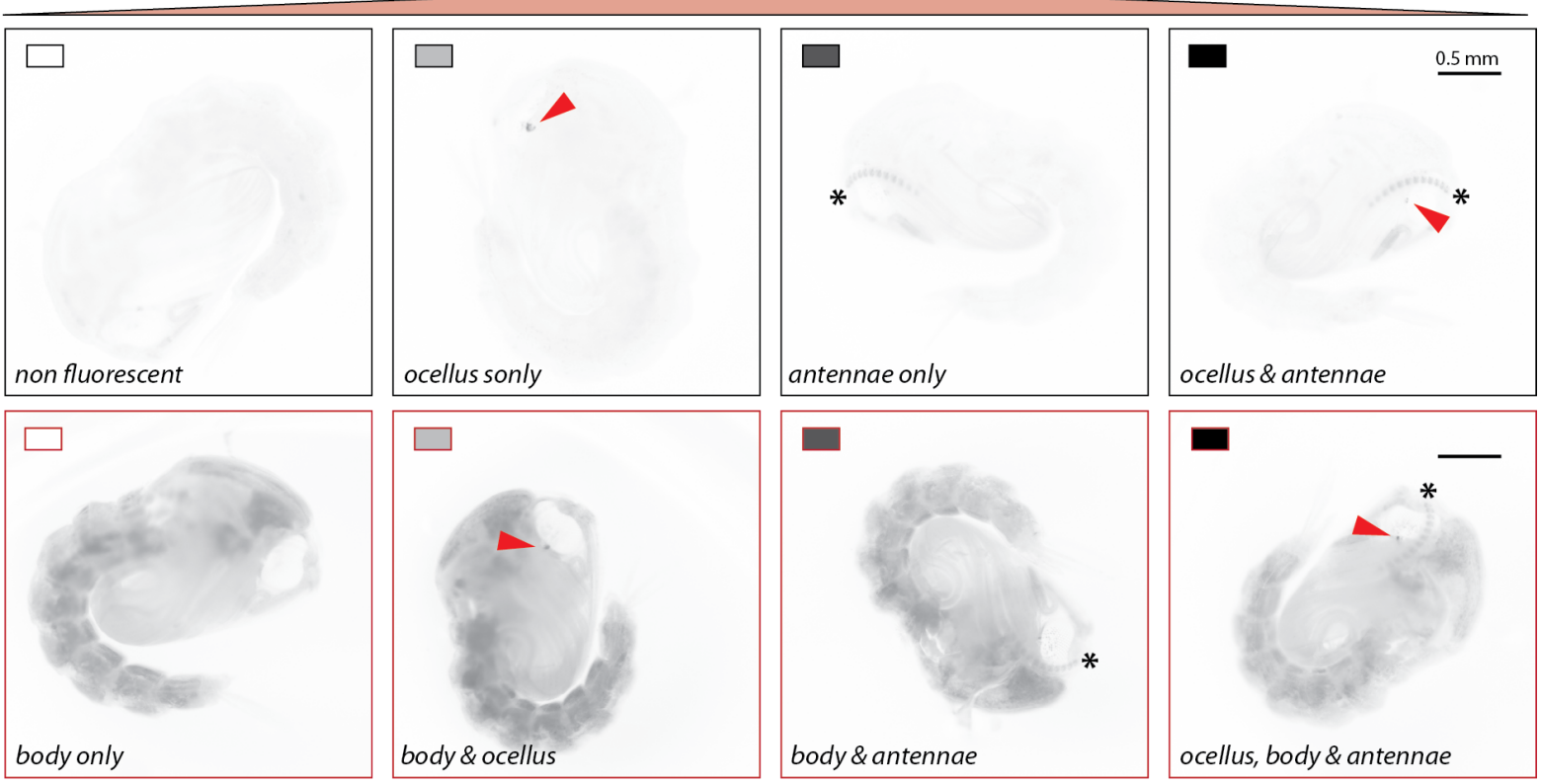

ocellus \& antennae

B $\square$ female $\square$ male

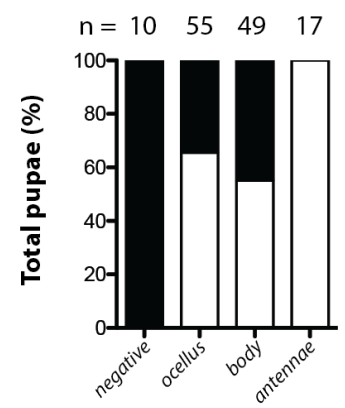

C

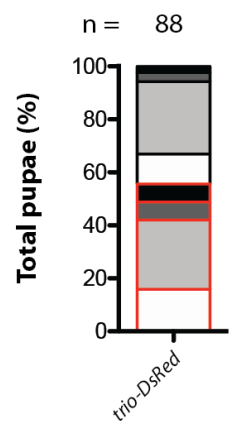

D $\square$ lowfluo $\square$ fluo

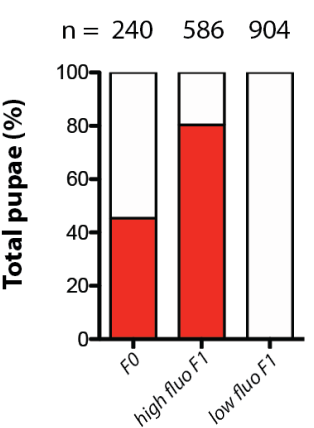

E
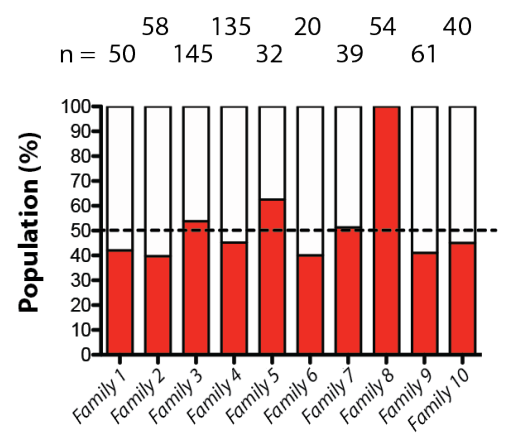

Figure S6. trio-DsRed pupae display high variability in DsRed expression.

A) Representative images of DsRed expression patterns in trio-DsRed pupae. A subpopulation of pupae displayed high body DsRed expression (images in red frames) while remaining pupae showed no DsRed body expression (black frames). Some pupae displayed fluorescent ocelli and/or fluorescent antennae. The presence of DsRed expression in body, ocelli and antennae occurred in different combinations that are represented by rectangles with different shades and frames. Scale bar: $0.5 \mathrm{~mm}$ B) Fluorescence patterns in pupae in relation to their sex. Non-fluorescent pupae were exclusively female while pupae with fluorescent antennae were exclusively male. Data pooled from four generations ( $\geq 20$ pupae per generation). C) Proportion of each fluorescence pattern in relation to the whole population. Shading and framing of the different patterns as in (A). D) Proportion of pupae with high and low body fluorescence in the trio-DsRed colony (F0) and after intercrossing individuals displaying high or low pupal body fluorescence (F1). The number of analyzed individuals is given above each column. E) Females hatched from pupae with high body fluorescence analyzed in (D, high fluo F1) were kept separately and crossed to wild type males (Ngousso). The progeny of single females was evaluated at the pupal stage for body fluorescence (same color code as $\mathrm{D}$, dashed line indicates $50 \%$ of the population). Females of families 5, 6, 7, 8 and 10 were also evaluated for salivary gland fluorescence which was present in all specimens. 

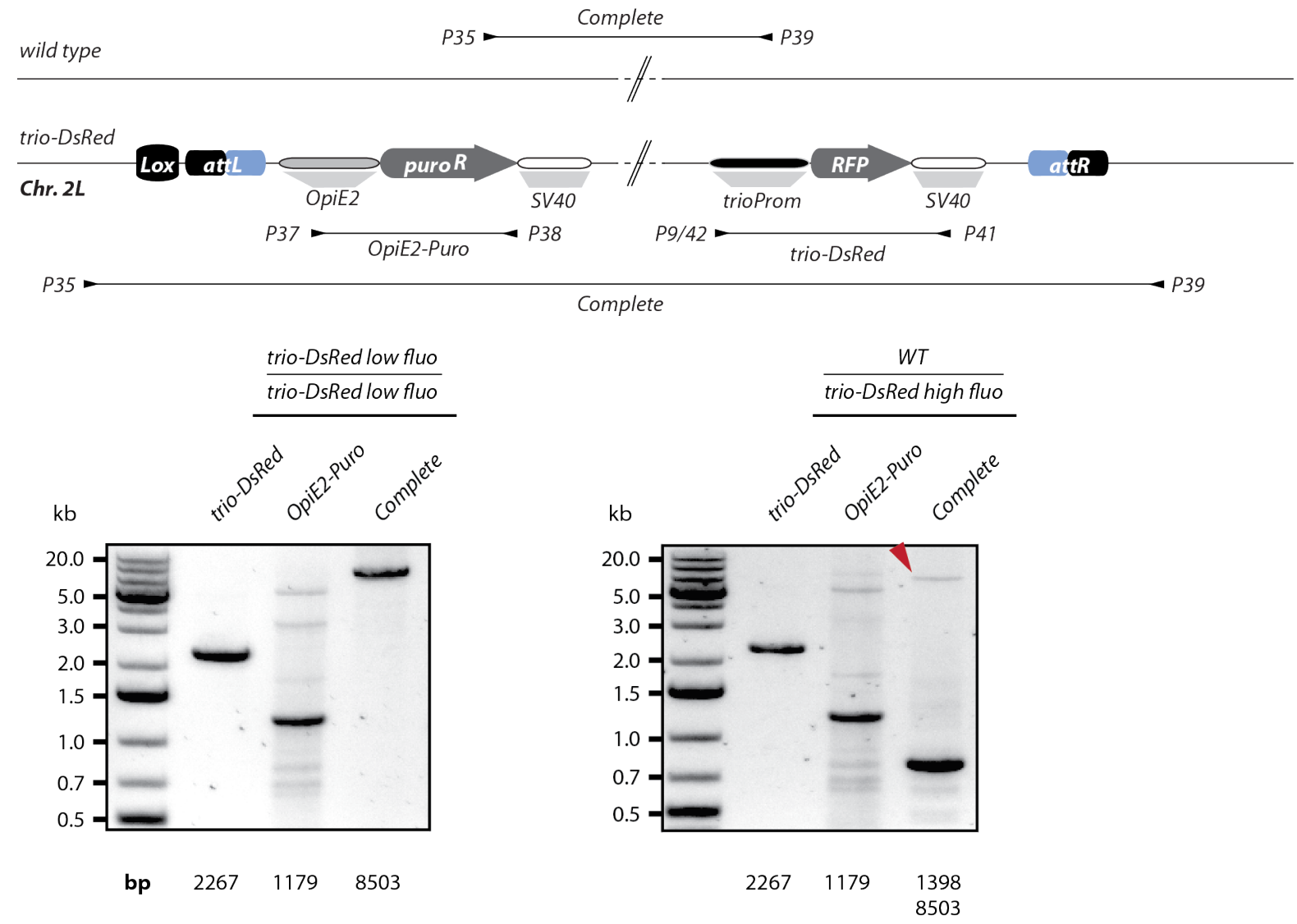

Figure S7. Genotyping of trio-DsRed high fluo in comparison to trio-DsRed low fluo.

Genotyping of a female mosquito homozygous for the trio-DsRed low fluorescence transgene taken from the colony generated by crossing fluorescence negative pupae (see Fig. S6D) in comparison to a female mosquito heterozygous for the trio-DsRed high fluorescence transgene and the wild type allele obtained from family 1 . An illustration of the modified X1 locus containing the trio-DsRed transgene in comparison to the unmodified wild type allele is shown on top. Genotyping primers and expected products are shown as black arrows and lines, respectively. The length of the expected PCR products is indicated below the gel images. Note that for the heterozygous female carrying the trio-DsRed high fluorescence transgene the smaller PCR fragment amplifying the unmodified locus dominates while the long PCR fragment representing the transgene containing allele is visible as a faint band at the upper edge (marked by a red arrow). The OpiE2-Puro PCR to verify the presence of the transgene gave several unspecific bands. PCRs were performed on genomic DNA obtained from single mosquitoes. 

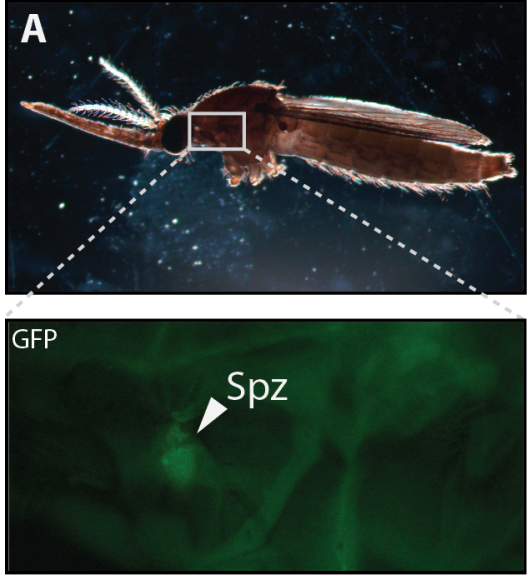

RFP

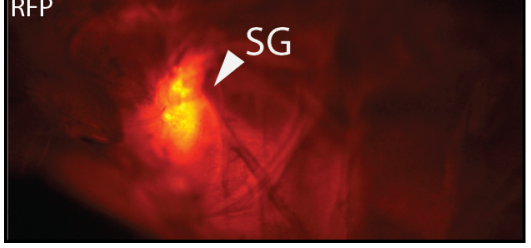

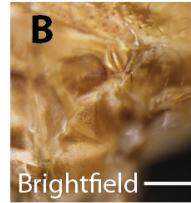
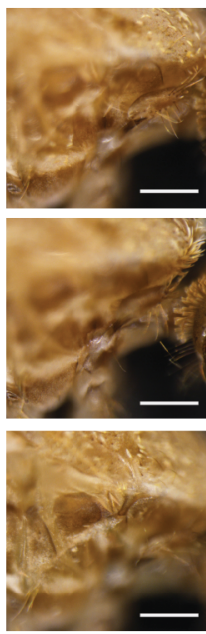
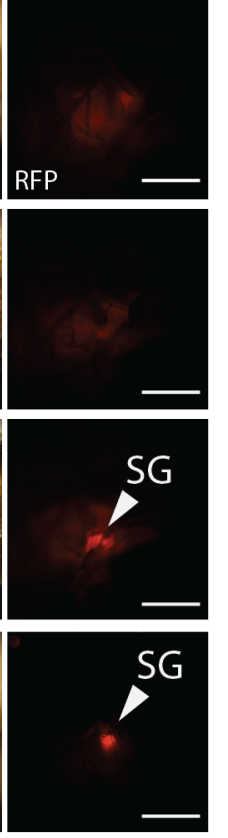

C yellow(-)-aapp-DsRed
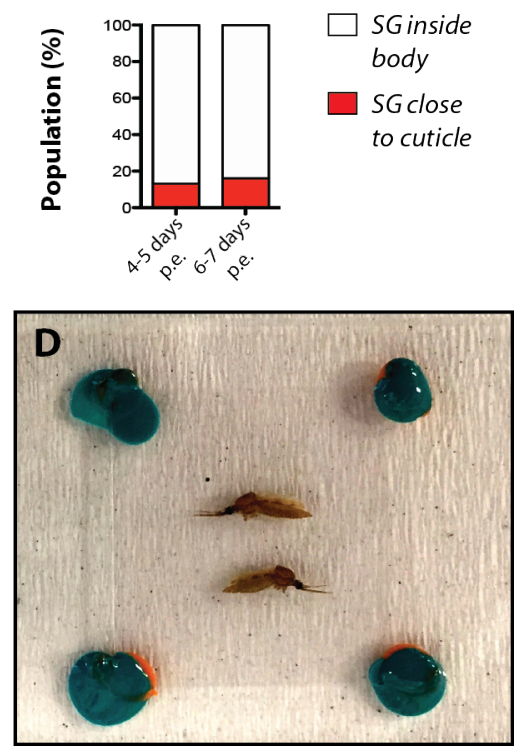

Figure S8. Selection and sample preparation of mosquitoes for in vivo imaging.

A) Images of an infected mosquito with sporozoites (Spz, GFP channel) inside salivary glands and salivary gland (SG, red channel) localization close to the cuticle. GFP and DsRed images show the mosquito neck region squared in the brightfield image (top). B) Positioning of the salivary gland varies between mosquitoes, either deep (rows 1 \& 2 ) or close to the cuticle (rows $3 \& 4$ ). Brightfield (left) and DsRed (right) images of the same field of view. Images were acquired with the same settings. Scale bar: $250 \mu \mathrm{m}$. C) Quantification of salivary gland positioning according to the fluorescence pattern shown in (B). Two mosquito batches were evaluated per time point post emergence (p.e.). The number of evaluated mosquitoes is indicated above each column. D) Sample preparation before imaging. Legless mosquitoes were glued on a microscopy slide and covered with a cover slip underlaid with orange plasticine to avoid squeezing. Corner areas were subsequently coated with teal-colored nail polish to prevent the cover slip from moving. 
1018 Movie 1. Sporozoites display active motility inside the mosquito.

1019 Movie showing three sporozoites imaged in close proximity to the salivary gland. Sporozoites appear to be attached to a larger vessel and observed movements might be a mixture of active motility influenced by passive hemolymph flow. Imaging performed with a Hamamatsu Orca Flash 4.0 V1 camera using a $63 x$ (NA 1.4) objective. GFP signal of a 3 min movie with 3 seconds between frames.

\section{Movie 2. Sporozoites form accumulations close to the salivary gland.}

Movie showing an accumulation of sporozoites close to the salivary gland directly below the cuticle. Several sporozoites display active back and forth movements. Imaging was performed with a Yokogawa CSU X-1 camera using a 63x (NA 1.4) objective. The left side of the movie displays the DsRed signal (salivary gland) while the right side shows the GFP signal (sporozoites) in black on white. Two min movie with 2 seconds between frames.

\section{Movie 3. Sporozoite crawls through tissue by employing back and forth gliding.}

Movie showing an actively moving sporozoite below the cuticle. The sporozoite was captured in proximity to the gland. By actively moving back and forth the sporozoite crawls slowly forward reaching eventually deeper tissues layers. Imaging was performed with a Yokogawa CSU X-1 camera using a 63x (NA 1.4) objective. The left side of the movie displays the DsRed signal (salivary gland) while the right side shows the GFP signal (sporozoites) in black on white. Two min movie with 2 seconds between frames. 
1040

1041

1042

1043

1044

1045

1046

1047

1048

1049

1050

1051

1052

1053

1054

1055

1056

1057

1058

1059

1060

1061

1062

1063

1064

1065

1066

1067

1068

1069

\section{Supplementary Table S1.}

List of primers used for cloning plasmids to generate salivary gland reporter lines as well as yellow(-)KI and sag(-)KI lines. Nucleotides highlighted in green represent the cutting site for $B s a \mathrm{I}$. The guanin written in white on a black background indicates an undefined position of the $B s a \mathrm{I}$ cutting site which can be any nucleotide. The nucleotides marked in red represent the overhangs needed for assembly of the GoldenGate building blocks. Sequences written in black define primer binding sites and nucleotides highlighted in orange represent overhangs to allow fusion of DNA sequences by assembly PCR.

Primer Sequence (5' to 3')

P1 GGTCTCGATCCCTTTTCTTTTACCCTTTGT

AACACGCTAATAACG

P2 GGTCTCGCTCTGTCGTTTTTTTGTTGTTGTG

GTATTATTTCACTG

P3

GGTCTCGAGAGATGGCTCAAGAGTTTGTG

AACTGCAAAATC

P4

GGTCTCGAAGCTTACTTGTACAGCTCGTC

CATGCCG

P5

GGTCTCGAGAGATGGTGCGCTCCTCCAAG

AACG

P6

GGTCTCGAAGCCTACAGGAACAGGTGGT

GGCGGC

P7

GGTCTCGATCCTTTTCTGAGGTGATCTTTC

GAAAAGATCG

P8

GGTCTCGCTCTGTAACTGGGAGCAGATTG ATTTTATGG

P9

GGTCTCGATCCCTTTCATGCGTACAATGTG TATGATCACTG

P10

\section{Description}

aapp_5'UTR_forward

aapp_5'UTR_reverse

hGrx1-roGFP2_forward

hGrx1-roGFP2_reverse

DsRed_aapp/trio_forward

DsRed_aapp/trio_reverse

trio_5'UTR_forward

trio_5'UTR_reverse

saglin_5'UTR_forward

saglin_5'UTR_reverse 
AAACAGACGAACAGCTTGTTCTCC

1081

P19

1082

1083

1084

1085

1086

1087

1088

1089

1090

1091

1092

P11

P12

P13

CCTTGTAGCGTGCCTGGTGGCCGC

CCTTGGCGACTACGTGCCCACGAA

AAACTTCGTGGGCACGTAGTCGCC

CCTTGGAGAACAAGCTGTTCGTCT

P20 AACGCTTTCCCTGCAGCGTAC

P23 CCTTGCGTTCAAGTCATCTCGAGC

P25 CCTTGCCGCAGTCCTCGCGGCTGG

P27

CCTTGAAGTTTGCAACGTTTGCGA

P28 AAACTCGCAAACGTTGCAAACTTC

P29
AAACGCGGCCACCAGGCACGCTAC

GGTCTCGAACAGAATTGAACGCCCAAGGTACGGCTG

GGTCTCACCCCGCTTCTGCCACTAAGGAACTTCTGTG

P21 GGTCtCGAAGACGTGTGTCCTGGAaGtGTGATTTATCG Yellow_3'UTR_fw

P22 GGTCTCGAAGCGTAGCGTGCCTGGTGGCCGCCGG

P24 AAACGCTCGAGATGACTTGAACGC

P26 AAACCCAGCCGCGAGGACTGCGGC
DsRed_saglin_forward

DsRed_saglin_reverse

Yellow_gRNA1_fw

Yellow_gRNA1_rev

Yellow_gRNA2_fw

Yellow_gRNA2_rev

Yellow_gRNA3_fw

Yellow_gRNA3_rev

Yellow_5'UTR_fw

Yellow_5'UTR_rev

Yellow_3'UTR_rev

Saglin_gRNA1_fw

Saglin_gRNA1_rev

Saglin_gRNA2_fw

Saglin_gRNA2_rev

Saglin_gRNA3_fw

Saglin_gRNA3_rev

Saglin_5'UTR_fw 
P30 GGTCTCAATGGTTATAAGTGAACCTTATGCAAACCTTCCCTG Saglin_5'UTR_rev

1094

P31

GGTCTCGAAGAGCGTCGGCGACGGTCTAAAAG

Saglin_3'UTR_fw

1095

P32

GGTCTCGAAGCGTTCAAGTCATCTCGAGCCGG

CTTCTTCTGCTGCCGCAGAAAC

Saglin_3'UTR_rev

1097 
1098

1099

1100

1101

1102

1103

1104

1105

1106

1107

1108

1109

1110

1111

1112

1113

1114

1115

1116

1117

1118

1119

1120

1121

1122

1123

1124

1125

1126 P48 CTGTCCCTTCAGCTTCACCAGCTC

1127

Supplementary Table S2. also been used for analytical PCRs.

Primer Sequence (5' to 3')
P47

GCTTATAAGAGCGCTACGGGTGTAC

P33 GGTCTCGAACAGAATTGAACGCCCAAGGT

ACGGCTG

P34 GGTCTCACCCCGCTTCTGCCACTAAGGAAC TTCTGTG

P35 CCCGTCATGAGTTCAAGCCTGAA

P36 CCCGGTCTCAAATTGCCCGCCGTGACCGTC GA

P37 CAAGCACCTTTATACTCGGTGGCCTC

P38 TTCCAGGAAGGCGGGCACCCC

P39 ACTGCAACCCATTCACACTG

P40

P41

CAGGGAAGGTTTGCATAAGGTTCACTTATA

AATGGTGCGCTCCTCCAAGAACG

P42 GGTCTCGATCCCTTTCATGCGTACAATGTGT ATGATCACTG

P43 CCTTGAAGATTGGAATCCATCCAT

P44 GGTCTCGATCCCTTTTCTTTTACCCTTTGTAAC ACGCTAATAACG

P45 GGTCTCGATCCTTTTCTGAGGTGATCTTTCGA

AAAGATCG

P46

GGTCTCGAGAGATGGCTCAAGAGTTTGTGAA

CTGCAAAATC

P49 GAACGACAGGTGAGCTGACAGTAAC

List of primers used for genotyping. Note that some primers used for construct cloning have

Binding site

yellow_5'_forward

yellow_5'_reverse

X1_forward_1

attB_reverse

OpiE2_forward

PuroR_reverse_1

X1_reverse

DsRed_forward

SV40_reverse

sag_forward

X1_forward_2

aapp_forward

trio_forward

hGrX1-roGFP2_forward

Sag_5'_fw_genotyping

Sag_3'_rev_genotyping

Yellow_5'_fw_genotyping 
1128 P50 GATTTGGCGAGATGGTTTGTGCGAC

1129 P51 GAGCTCGAGTTCAGGCACCGGGCTTGC

$1130 \quad$ P52 CTTCGGGCATGGCGGACTTG

1131

P53

PuroR_reverse_2

EGFP_reverse

1132 


\section{Supplementary File S1.}

1134 Promoter sequences of the trio, saglin and aapp genes used to generate the mosquito lines 1135 aapp-DsRed, aapp-hGrx1-roGFP2, sag-DsRed and trio-DsRed. Nucleotides highlighted in 1136 red are part of the primer sequence used to amplify the promoter sequence. Sequences with a 1137 1138 grey background indicate the 5'UTRs of the trio and aapp transcript, respectively.

\section{aapp promoter sequence (AGAP009974, 1673 bp):}

СTTTTCTTTTACCСTTTGTAACACGCTAATAACGAAAGACCTCCTGCGTGTTAGTACG TGGTGAGGATGATGCGTTTTAGCCTAGCTAGTATTCAAAAAACCTATTAATGCAACAATTAC AGTGTGAGGTTACAAAATGGAATTAATTAAACACACTGCTCAACACAGCCATCGTGCTGCAT CGTGCTTTGTTGAGGCGCAACAGTTAAATTGTAAGTGCAACCACCGGTATAGAGAGCTGCTT TTGGCAAATGATATGGAATTGTGCTAAATTATGTAATGTTTTTTTTAACTAACTCGTTTACC CGTTAATGGTGGAATGTTTGCAGGTGCGTAGATTAATTTTTTTTAAATGTGCAAATGGAGAA AACAGGATAACGCAAAAGTATTTCCTTCGCTGTAGTAATAGGCTATTATTTATGTACGTCTT TATGTGACCCCGCTAGTGTTGGCTGTATTTTATATCTGTAGGTAAATGACGCGCTATGTATT GTAATAAAATTGGACAAACATTTGCATGTACGATCTGTTAGCTTAGAGAATAAGTATTTTTC TTTTTCGCTACATTTCTCTAAATTCGACCGACGATGACTGTTTTAGGTGTTTTTTCGTAACT TGATCAAGGTAACACCTTTTCTTGGCGTAACGTCCTACGCGGACATGCCGGCCGGCCTTATT CAGGCTTTCGAGACTTAATTCATTACCACGTAGCCGGATAAGTCCAATCCTTGCACAACGGG GGAACGGGTGTCCATTTCAGGCTTGGGCCCATGACGGGCATGTTATTGAGTCGTTCGAGTTG ACGACTGTACCATGGGACCGCCCCATTTTTGGCAGTCCATTGATCGTTACGTCGTTTTACGT CTACTGAAGGATGTCCGTTATCAGGTCAAGTCCAGCATAGATTAAGTCTCCCATACGAAGGA TTGATTAGCTCGAGACTTAATGTTATATACAAAATTCTTATACATGTTCTAGATCAGGATTG TTGCGCTACACTGTCTTCCATGTCGATCTTCTATATTCAGTTTTTGGCACAGTGATCGTCAC TGTAATCTCAATAGATTTGAATTTCAATACTTCTATGGGAGGAGGATGAGTAGAAAGGTGTC CAAAGCGCTGGATCTATGGCTGCAACTCCTCATGCTTGTAAGCACCCGACAGCCAATTCGAT CTACCTGACCTGATGCGGTATTCGAACTAAAAGCACAGATCAAGCACTGTTTCACACGATCT GAAGCACTGTTCTACTGCAAAGATAAGTGCATATTAGTTATTTTTCATACCGATCTTCACTG CGACGGTTTAGCGATCTTCATTATTTTCCACCTTCACCCCTCAACCCAGGGAGTGCGCTTTT CCCCGTCGAGATAAAATTACTAAATGTAAACTTTGCATGCAATTATGTAGCTGTATGGTAAG CAAATAAAAATCTTTCCTCCTATTGCAACACGTCGCTTGTTTGCAGCGGATATTTGCCCACA GTAAAGCCCGATCGCCTGTGCATTGTGTTTAACACGTGCTGCAAGCTGTATCAATACCGATC GATTGTATGATTCCACGATCTTCGTGTGGTATAAAAACGGCGCTGAACAGCAGTAAAGATCA TCACTTACATTTGTTTTATTCTCCACGGAGTCAGTGAAATAATACCACAACAACAAAAAAAC 
trio promoter sequence (AGAP001374, 1537 bp):

TTTTCTGAGGTGATCTTTCGAAAAGATCGATCGACCGACCCCCAACGGCAACTCTCCC TCGGATGACCTCAGCCCAGAATAGGCATTCTTTCAGCAAGTCATTCTATCATCCCTTTTACA TCTTACGTTACTCCAAAAAAAGAGAAGCTTTCCACCCCAAAGCTTTCGTTGTGGTGCAGCTT TCGATGGTGATCGCGATCACGGTACCACACGTACGTTTGTTTGCTACTCTCGCTCACCGTTT TCTTCGATTTCGTGTCTCGGGTACCGTTTGCTCGGTGCGGTAGTGCTGCGAAGAAAGCCGTT GGCCGAGAGAATAATGCTCACTTCGGCTGCACGCAACTCGCCGTGCCACAGCCGTGGAAGGG GTAGGTTGGATGGTCGGGGTCGGGACCGCAACGCCGAAGTGAACGCCGGCGTGCCAGCGAAC GATCGCACCCCGGCACAGTCCAACTGCGTCGCTGGGCGCGCTCGGCGGACGGTTCTCGGTGT GTGCGCCCCCTTTGGTGCTCATCCCTCATATCTCATCTCATCCTTTCGCGCTGCTCGCGGTT CCCGCAGAACCACGCGCGAGAGGGAGTTGCGCGAACCAGGGAGAGCCCGACACCTGGGCAGC GTCCAGGCGGACCTGTTCGGGAACGGTCGCGGTGTATAAAACTCGATTCCCGCCCGCCGTTA GGTAACAGTCCATTGTGTAACGTTGCCGGGATCGCGAGCCATCCTGCAGGAACACAGCCGTG CGAGTTTGTGTGCGCGTGTATTTTGCCTACAAGCAAGTGAGCAGCAATCAGTGAGAACAACA TCTAAAACAGAAAAACAAACAGTGAACTACAGTGCTGTGGAGCAGTGGAAATTGGTGTTAAT TGAAGAGCTTTGTACATCGATCACACGGACAACCACGCACGTGGAGAAAGTTCTGCTGCGGT GCGGGTTACTTGCGTACACCACATTAAACATTGTGTGTGGCATTTAAAATAAATATACTCGC GCССTCCССTCAGCTGCTCTCGCGGGCACGCCCGATGCCAGAGGTCGCCCCGACCGGACATT GAACGTGGAAAGAAGAAAGTTGTCGGTTGTTTACACTTCCGTCCTGAGTGGTGTTGTGGCTA CCGTTTGTGACCGTTGGTGAAATAAAGTAACATACCCCTCCCGAGGGCAGCAGCGACAATGA CCTCCGGAAATTCGATAACAATACGTAAGTTACTTTCATTTTTTGCAAATTAAATTACCTCG TGATAATTGAGTGGAGTGAGTAGGTACAGGTTGTTAACGCGTCTTTTGTTTAGTTCGGCAAT CCGGATCCGAAGATGAGAGGAGATTGCCTCTGATGATGATGTTATCATGATTGCTTGTGTAA CGACGCAAAGATTCTCAGAGAACAACTTCTCCCAACATAATTAATCTTGTTGAAGGGTCTGT AAACACGTGGTGGGATATCTTACAACTGAACCAATATTCGACCGATAGTATAAAGTCCTTCG CACCATCGATGCGTTTACCATTTTCCCATAAAATCAATCTGCTCCCAGTTACA

sag promoter sequence (AGAP000610, 220 bp):

CTTTCATGCGTACAATGTGTATGATCACTGATGACAAAGCGCTTGGGTGGGCGCTTGC TGCTGAAGCGTGGCTGACAAATGGGGGGGGGGGGGGCTTGAACAAATATTTGCACACACGAT AATGCTCTTGTTTGCCATGCGCTGACGGAAGACAAATCGCTGCAAGCGACGGCTCAAACCTG CTCAAACCAGGGAAGGTTTGCATAAGGTTCACTTATAA 